\title{
High-resolution X-ray spectroscopy of T Tauri stars in the Taurus-Auriga complex
}

\author{
A. Telleschi ${ }^{1}$, M. Güdel ${ }^{1}$, K. R. Briggs ${ }^{1}$, M. Audard ${ }^{2}$, and L. Scelsi ${ }^{3}$ \\ 1 Paul Scherrer Institut, Würenlingen and Villigen, 5232 Villigen PSI, Switzerland \\ e-mail: atellesc@astro.phys.ethz.ch \\ 2 Columbia Astrophysics Laboratory, Mail code 5247, 550 West 120th Street, New York, NY 10027, USA* \\ 3 Dipartimento di Scienze Fisiche ed Astronomiche, Sezione di Astronomia, Università di Palermo, Piazza del Parlamento 1, \\ 90134 Palermo, Italy
}

Received 5 August 2006 / Accepted 30 October 2006

\section{ABSTRACT}

\begin{abstract}
Context. Differences have been reported between the X-ray emission of accreting and non-accreting stars. Some observations have suggested that accretion shocks could be responsible for part of the X-ray emission in classical T Tauri stars (CTTS).

Aims. We present high-resolution X-ray spectroscopy for nine pre-main sequence stars in order to test the proposed spectroscopic differences between accreting and non-accreting pre-main sequence stars.

Methods. We used X-ray spectroscopy from the XMM-Newton Reflection Grating Spectrometers and the EPIC instruments. We interpret the spectra using optically thin thermal models with variable abundances, together with an absorption column density. For $\mathrm{BP}$ Tau and $\mathrm{AB}$ Aur we derive electron densities from the O VII triplets.

Results. Using the $\mathrm{O}$ VII/O VIII count ratios as a diagnostic for cool plasma, we find that CTTS display a soft excess (with equivalent electron temperatures of $\approx 2.5-3 \mathrm{MK}$ ) when compared with WTTS or zero-age main-sequence stars. Although the O VII triplet in BP Tau is consistent with a high electron density $\left(3.4 \times 10^{11} \mathrm{~cm}^{-3}\right)$, we find low density for the accreting Herbig star AB Aur $\left(n_{\mathrm{e}}<10^{10} \mathrm{~cm}^{-3}\right)$. The element abundances of accreting and non-accreting stars are similar. The Ne abundance is found to be high (4-6 times the Fe abundance) in all K and M-type stars. In contrast, for the three G-type stars (SU Aur, HD 283572, and HP Tau/G2), we find an enhanced Fe abundance (0.4-0.8 times solar photospheric values) compared to later-type stars.

Conclusions. Adding the results from our sample to former high-resolution studies of $\mathrm{T}$ Tauri stars, we find a soft excess in all accreting stars, but in none of the non-accretors. On the other hand, high electron density and high $\mathrm{Ne} / \mathrm{Fe}$ abundance ratios do not seem to be present in all accreting pre-main sequence stars.
\end{abstract}

Key words. stars: coronae - stars: formation - stars: pre-main sequence $-\mathrm{X}$-rays: stars - stars: flare

\section{Introduction}

Young, late-type stars are known to be luminous X-ray emitters. This is particularly true for $\mathrm{T}$ Tauri stars, which are divided into two classes: the classical T Tauri stars (CTTS) and the weakline T Tauri stars (WTTS). The CTTS display strong $\mathrm{H} \alpha$ emission lines as a signature of accretion and an infrared excess as a signature of the presence of a circumstellar disk. The WTTS display much weaker $\mathrm{H} \alpha$ lines and no infrared excess, a sign that accretion has ceased and a thick disk is no longer present. While CTTS are thought to be in an earlier evolutionary stage, both types of stars are simultaneously present in star forming regions.

X-rays from T Tauri stars were first detected in early studies with the Einstein satellite (Feigelson \& DeCampli 1981) and surveyed more extensively with the ROSAT satellite (e.g., Feigelson et al. 1993; Neuhäuser et al. 1995). Both types of young stars exhibit variability on time scales of hours to days. Their overall X-ray properties were found to be similar to the X-ray

* New address (since September 2006): Integral Science Data Centre, Ch. d'Ecogia 16, 1290 Versoix, Switzerland \& Geneva Observatory, University of Geneva, Ch. des Maillettes 51, 1290 Sauverny, Switzerland. characteristics of main-sequence stars, and the X-ray emission was interpreted to arise from a corona.

However, most young stellar objects, especially in their early evolutionary stage, are believed to be fully convective, therefore the generation of magnetic fields through a solar-like dynamo should not be possible. Also, the presence of a disk and accretion in CTTS adds another important element: shocks generated by accretion of circumstellar material onto the star could contribute to the X-ray emission.

The influence of accretion on X-ray emission processes has been debated. In the ROSAT observations of the Taurus-Auriga complex, Stelzer \& Neuhäuser (2001) found that the $L_{\mathrm{X}} / L_{*}$ ratio (where $L_{*}$ is the bolometric luminosity of the star) is lower in CTTS than in WTTS. This has been confirmed in the recent, more comprehensive XMM-Newton Extended Survey of the Taurus molecular cloud (XEST), but on the other hand, no direct correlation has been found between accretion rate and $L_{\mathrm{X}} / L_{*}$ (Telleschi et al. 2007). Similar results have also been reported from the Chandra Orion Ultradeep Project (COUP), where the CTTS are found to be less luminous in X-rays than WTTS (Preibisch et al. 2005). In both surveys, the bulk X-ray emission is consistent with coronal emission. 
On the other hand, recent results from the high-resolution $\mathrm{X}$-ray spectroscopy of some CTTS seem to indicate the presence of X-ray emission generated in accretion shocks. The most evident case is TW Hya. The Chandra and XMM-Newton spectra of TW Hya (Kastner et al. 2002; Stelzer \& Schmitt 2004) are dominated by emission from plasma at temperatures of $\approx 3 \mathrm{MK}$, which is much lower than usually found in T Tauri stars. The O VII triplet, consisting of lines at $21.6 \AA$ (resonance, $r$ line), $21.8 \AA$ (intercombination, $i$ line), and $22.1 \AA$ (forbidden, $f$ line), displays an $f / i$ flux ratio much below unity. This triplet is density-sensitive (Gabriel \& Jordan 1969); in late type stars, typical values for the $f / i$ ratio are higher than unity (Ness et al. 2004; Testa et al. 2004), indicating densities of at most a few times $10^{10} \mathrm{~cm}^{-3}$. In TW Hya, the measured electron density exceeds $10^{12} \mathrm{~cm}^{-3}$ (Kastner et al. 2002; Stelzer \& Schmitt 2004); i.e., it is at least two orders of magnitude higher than typical coronal densities. Studies of other density-sensitive lines lead to similar results: the He-like Ne IX triplet in the Chandra observations gives $\log n_{\mathrm{e}}=12.75$ (Kastner et al. 2002), while a study by Ness \& Schmitt (2005) presents additional evidence of high density from the flux ratios of Fe XVII lines. Further, Kastner et al. (2002) and Stelzer \& Schmitt (2004) find an abundance anomaly in the spectrum of TW Hya, with certain metals being underabundant with respect to the solar photospheric abundances, while nitrogen and neon are found to be strongly overabundant. This anomaly was interpreted by Stelzer \& Schmitt (2004) as being due either to metal depletion by condensation onto grains in the accretion disk or to an abundance anomaly present in the original molecular cloud. A particularly high $\mathrm{Ne} / \mathrm{Fe}$ abundance ratio was also found by Argiroffi et al. (2005) for TWA 5, a stellar system in the TW Hya association. This object is a quadruple system and one of the components could be an accreting star (Mohanty et al. 2003), although it is not possible to identify which of the stars is the X-ray source. The high $\mathrm{Ne} / \mathrm{Fe}$ ratio could be due to the accreting star, but could also be an environmental effect of the TW Hydrae association (Argiroffi et al. 2005). Drake et al. (2005) have proposed to use the Ne/O abundance ratio as a diagnostic for metal depletion in accreting stars. This ratio is in fact found to be substantially higher in TW Hya than in all other studied stars (also higher than in TWA 5, another star of the TW Hydrae association).

Schmitt et al. (2005) discuss the high-resolution X-ray spectrum of the CTTS BP Tau observed with XMM-Newton. This spectrum also displays an $f / i$ ratio lower than unity, resulting in an electron density of $\log n_{\mathrm{e}} \approx 11.5$. However, the BP Tau spectrum is dominated by a hot plasma component. The authors interpret the hotter component as originating in a corona or from magnetic interaction between disk and corona, while the soft component may originate in accretion shocks.

Robrade \& Schmitt (2006) present a comparative study of the high-resolution spectra of four pre-main sequence accreting stars: BP Tau, CR Cha, SU Aur, and TW Hya. They tentatively added CR Cha as another CTTS with a low O VII $f / i$ ratio, but the low signal-to-noise ratio $(\mathrm{S} / \mathrm{N})$ makes the measurements uncertain. The O VII triplet was not detected in SU Aur. The $\mathrm{Ne}$ abundance is found to be enhanced relative to $\mathrm{Fe}$ also in $\mathrm{CR}$ Cha and BP Tau. However, the $\mathrm{Ne} / \mathrm{O}$ abundance ratio of $\mathrm{BP}$ Tau is similar to the ratio found for other (non-accreting) stars and not as high as that of TW Hya, which may be due to evolutionary effects in the accretion disk (Drake et al. 2005). Recently, Günther et al. (2006) studied the Chandra spectrum of another CTTS, V4046 Sgr, and measured a high O VII $f / i$ ratio, consistent with electron densities of $\log n_{\mathrm{e}} \approx 11.5$.
High-resolution spectroscopy is of crucial importance for identifying the soft component in X-ray spectra of young premain-sequence stars because emission lines formed at low coronal temperatures can be accessed, such as lines of $\mathrm{C} \mathrm{V}, \mathrm{C} \mathrm{VI}$, $\mathrm{N}$ VI, N VII, O VII, and O VIII, whereas the energy resolution of CCDs is insufficient for revealing these lines individually. The possibility of measuring density-sensitive lines is also essential for distinguishing between X-ray emission that may originate in accretion shocks and coronal emission. A comparative study of the X-ray spectra of CTTS and WTTS should further our understanding of the role of accretion in the production of X-ray emission.

In this paper we present high-resolution X-ray spectra of four CTTS, four WTTS, and one Herbig Ae/Be star. These observations are part of the XEST project (Güdel et al. 2007a), the $X M M$-Newton survey of the most populated regions in the Taurus molecular cloud (TMC). The purpose of that survey is to study $\mathrm{X}$-ray emission in a large fraction of the TMC population. While the spectra of HD 283572, SU Aur, and BP Tau have been extracted from archival data and already been presented in the literature (Scelsi et al. 2005; Schmitt et al. 2005; Robrade \& Schmitt 2006), the RGS spectra of the other stars are shown for the first time in this paper. We analyze the spectra of the former three stars again to provide a consistent comparison.

The structure of our paper is as follows. The stellar sample is described in Sect. 2, while we present our observations and data reduction in Sect. 3. The results are presented in Sect. 4, while a detailed discussion of the $\mathrm{O}$ VII triplets is presented in Sect. 5. In Sect. 6 we discuss our results and Sect. 7 contains our conclusions.

\section{Stellar sample}

We present RGS spectra of 9 pre-main sequence stars. Four of them are CTTS, four are WTTS, and one is an intermediate-mass young star belonging to the class of Herbig stars (Herbig 1960). The principal properties of the stars are listed in Table 1, but we discuss some specific characteristics below. In Table $1, L_{*}$ is the stellar (photospheric) bolometric luminosity, $M$ is the mass, $A_{\mathrm{V}}$ and $A_{\mathrm{J}}$ are the extinctions in the $V$ and $J$ bands, respectively, $P_{\text {rot }}$ is the rotation period, $E W(\mathrm{H} \alpha)$ is the equivalent width of the $\mathrm{H} \alpha$ line (positive for emission), and $\dot{M}$ is the mass accretion rate. If not otherwise noted, these properties are reported from Güdel et al. (2007a) and references therein.

HD 283572 is a single G-type star that does not show signs of accretion and is therefore classified as a WTTS (Kenyon et al. 1998). Also, there is no evidence of an infrared excess that would be due to a circumstellar dust disk (Furlan et al. 2006). Favata et al. (1998) studied its X-ray variability, analyzing data from Einstein, ASCA, ROSAT, and SAX. Results of the XMM-Newton data set have already been presented by Scelsi et al. (2005).

V773 Tau is a system composed of four stars. The system was detected to be a binary by Ghez et al. (1993) and Leinert et al. (1993); the primary component was then discovered to be a binary itself (Welty 1995). Recently, a fourth component was detected by Duchêne et al. (2003). These authors show that the two close components $\mathrm{A}$ and $\mathrm{B}$ reveal properties of WTTS, while the C component shows a near infrared excess typical of CTTS. The fourth component has been classified as an infrared companion (IRC). The nature of IRC is still debated: they could be deeply embedded TTS undergoing strong accretion (see e.g., Koresko et al. 1997) or they may be embedded protostars (e.g., Ressler \& Barsony 2001). In the latter case, the four stars would be in three different 
Table 1. Properties of target stars.

\begin{tabular}{|c|c|c|c|c|c|c|c|c|c|c|c|c|}
\hline Star & XEST No. ${ }^{a}$ & $\begin{array}{c}L_{*} \\
\left(L_{\odot}\right)\end{array}$ & $\begin{array}{c}M \\
\left(M_{\odot}\right)\end{array}$ & $\begin{array}{l}\text { Age } \\
(\mathrm{Myr})\end{array}$ & $\begin{array}{c}R \\
\left(R_{\odot}\right)\end{array}$ & $\begin{array}{c}A_{\mathrm{V}} \\
(\mathrm{mag})\end{array}$ & $\begin{array}{c}A_{\mathrm{J}} \\
(\mathrm{mag})\end{array}$ & $\begin{array}{l}P_{\text {rot }} \\
\text { (d) }\end{array}$ & Spec. & $\begin{array}{c}E W(\mathrm{H} \alpha) \\
(\AA)\end{array}$ & $\begin{array}{c}\log \dot{M} \\
\left(M_{\odot} \mathrm{yr}^{-1}\right)\end{array}$ & $\begin{array}{l}\text { Type } \\
\text { TTS }\end{array}$ \\
\hline HD 283572 & $21-039$ & 6.50 & 1.70 & 7.92 & 2.56 & 0.38 & 0.11 & 1.55 & G5 & 0 & - & $\overline{\mathrm{W}}$ \\
\hline V773 Tau & $20-042$ & 5.60 & 1.53 & 6.35 & 1.91 & 1.39 & 0.31 & 3.43 & $\mathrm{~K} 2$ & $4-10$ & $<-10$ & $\mathrm{~W}$ \\
\hline $\mathrm{V} 410 \mathrm{Tau}^{b}$ & $23-032 / 24-028$ & 2.20 & 1.51 & 2.74 & 2.31 & 0.67 & 0.00 & 1.94 & K4 & $2-3$ & $<-8.8$ & W \\
\hline HP Tau/G2 & $08-051$ & 6.50 & 1.58 & 10.5 & 2.34 & 2.08 & 0.66 & 1.20 & G0 & $0-5$ & - & W \\
\hline SU Aur & $26-067$ & 9.90 & 1.91 & 6.02 & 3.06 & 0.90 & 0.21 & 1.70 & G2 & $2-6$ & $-8.30 /-8.20$ & $\mathrm{C}$ \\
\hline DH Tau & $15-040$ & 0.56 & 0.47 & 1.53 & 1.82 & 1.25 & 0.32 & 7.00 & M1 & $39-72$ & $-8.95 /-8.30$ & $\mathrm{C}$ \\
\hline BP Tau & $28-100$ & 0.95 & 0.75 & 1.91 & 1.97 & 0.49 & 0.14 & 7.60 & K7 & $40-92$ & $-7.88 /-7.54$ & $\mathrm{C}$ \\
\hline DN Tau & $12-040$ & 1.00 & 0.56 & 1.05 & 2.25 & 0.49 & 0.34 & 6.30 & M0 & $12-87$ & $-8.73 /-7.79$ & $\mathrm{C}$ \\
\hline AB Aur & $26-043$ & 49.0 & 2.70 & 4.0 & 2.31 & 0.25 & 0.24 & $<1.46$ & B9.5-A0 & $22-44$ & $\approx-8^{c}$ & $\mathrm{Ae}$ \\
\hline
\end{tabular}

${ }^{a}$ XEST catalog number: the first two digits define the exposure numbers, the last three digits are the source numbers (Güdel et al. 2007a).

${ }^{b}$ V410 Tau was observed in two different exposures, therefore two different XEST numbers are given (see below).

${ }^{c}$ From Telleschi et al. (2007) and references therein.

evolutionary phases. We refer the reader to Duchêne et al. (2003) for an exhaustive discussion. In this work we treat V773 Tau as a WTTS for two reasons: the primary and secondary stars (the most luminous components) are WTTS, and the hydrogen column density $\left(N_{\mathrm{H}}\right)$ found in our spectral fits $\left(2.0 \times 10^{21} \mathrm{~cm}^{-2}\right)$ is consistent with the optical extinctions found for the two WTTS $\left(A_{\mathrm{V}}=1.39 \mathrm{mag}\right)$ if a standard gas-to-dust ratio is assumed $\left(N_{\mathrm{H}} / A_{\mathrm{V}}=2 \times 10^{21} \mathrm{~cm}^{-2} \mathrm{mag}^{-1}\right.$; Vuong et al. 2003). The masses of V773 Tau A and B are 1.5 and $\approx 1 M_{\odot}$, respectively, while the mass of the $\mathrm{C}$ component is only $\approx 0.7 M_{\odot}$. The IRC would have a mass of $\leq 0.7 M_{\odot}$. Because the X-ray luminosities of TTS are correlated with mass (Preibisch et al. 2005; Telleschi et al. 2007), we expect the $\mathrm{A}+\mathrm{B}$ components to dominate the $\mathrm{X}$-ray spectrum. X-rays from V773 Tau were previously detected with ROSAT (Feigelson et al. 1994) and with ASCA (Skinner et al. 1997).

V410 Tau is a triple system (Ghez et al. 1993, 1997). V410 Tau A and B are separated by $0.12^{\prime \prime}$ with a much fainter $\mathrm{B}$ component. The $\mathrm{C}$ component is also much fainter than V410 Tau A. The primary has spectral type K4 with a mass of $1.5 M_{\odot}$ and an age of 2.74 Myr. V410 Tau was detected in X-rays by ROSAT (Strom \& Strom 1994), and more recently Stelzer et al. (2003) presented a set of Chandra observations of this source.

SU Aur is a single star of spectral type G2, with a mass of $1.9 M_{\odot}$. The properties of this object are described by DeWarf et al. (2003). Despite the low equivalent width (EW) of the $\mathrm{H} \alpha$ line reported in Table 1, its early spectral type and the measured infrared excess classifies SU Aur as a CTTS (Muzerolle et al. 2003a). The source is one of the X-ray-brightest CTTS and it has already been detected by Feigelson \& DeCampli (1981) with the Einstein Observatory and by Skinner \& Walter (1998) in an ASCA observation. Results from its XMM-Newton spectrum were recently published by Robrade \& Schmitt (2006, further analysis will be presented by Franciosini et al. 2007, in preparation).

HP Tau/G2 is a G0 star that forms a triple system with HP Tau/G3 (separation 10"), the latter itself being a binary (Richichi et al. 1994). The X-ray spectrum of HP Tau/G2 is contaminated by HP Tau/G3. However, fitting separate PSF in the EPIC data, we found that the source counts of HP Tau/G3 amount to only about $8 \%$ of the count rates of HP Tau/G2 (Güdel et al. 2007a). The contamination due to HP Tau/G3 is therefore negligible. Bipolar outflows have been detected from HP Tau/G2 (Duvert et al. 2000), although the star is classified as a WTTS.

DH Tau is another binary, the primary DH Tau A being a CTTS, with a mass of $0.5 M_{\odot}$ and an age of 1.5 Myr. DH Tau B is a brown dwarf (Itoh et al. 2005) with mass of 0.03-0.04 $M_{\odot}$ and an age of 3-10 Myr.

BP Tau is a CTTS of spectral type K7 with a mass of $0.75 M_{\odot}$ and an age of $\approx 2 \mathrm{Myr}$, according to the evolutionary model of Siess et al. (2000). The star has already been observed by the Einstein Observatory, with $L_{\mathrm{X}} \approx 1 \times 10^{30} \mathrm{erg} \mathrm{s}^{-1}$ (Walter \& Kuhi 1981). Results from the XMM-Newton spectrum have been discussed by Schmitt et al. (2005) and Robrade \& Schmitt (2006). They measured a relatively high density in the O VII triplet, suggesting that some of the X-rays could be produced in accretion shocks.

DN Tau is a CTTS of spectral type M0 with a mass of $0.56 M_{\odot}$ and an age of $\approx 1$ Myr. According to Muzerolle et al. (2003a), the inclination angle is $35^{\circ}$, and the disk is relatively small (0.05 AU in radius).

AB Aur is a Herbig star of spectral type B9.5-A0 with a mass of $2.7 M_{\odot}$ and an age of $\approx 4 \mathrm{Myr}$ (DeWarf \& Fitzpatrick, private communication). It is surrounded by a disk from which it is accreting material (see for example Catala et al. 1999), making it useful to compare the X-ray emission of this star with the X-ray emission of CTTS. A detailed discussion of the X-ray spectrum of this star is presented in Telleschi et al. (2007).

\section{Observations and data analysis}

The XEST campaign collected 28 XMM-Newton observations within the Taurus-Auriga complex (Güdel et al. 2007a). We obtained high-resolution X-ray spectra from nine targets within these fields of view, which are the focus of this paper. The observation log is given in Table 2. For each star, we used each spectrum from the two Reflection Grating Spectrometers (RGS, den Herder et al. 2001) and one of the spectra from the MOStype European Photon Imaging Cameras (EPIC, Turner et al. 2001). For HD 283572, the RGS1 instrument was out of operation and, therefore, we restricted our analysis to the RGS2 and MOS1 instruments. For all observations, the MOS instrument observed in "full frame" mode. Its detectors are sensitive in the energy range of $0.15-15.0 \mathrm{keV}$ with a spectral resolving power of $E / \Delta E=20-50$. The RGSs are suited to high-resolution spectroscopy, operating in the wavelength range of $6-35 \AA$ with a resolution of $\Delta \lambda \approx 60-76 \mathrm{~m} \AA$.

We reduced our data using the Science Analysis System (SAS) version 6.1. For the EPIC detectors, the data were reduced using the emchain task, and the sources were detected using the maximum likelihood algorithm emldetect (see Güdel et al. 2007a for more details). We reduced the RGS data using 
Table 2. Observation log.

\begin{tabular}{|c|c|c|c|c|c|c|c|}
\hline Stars & XEST No. & $\mathrm{ObsID}^{a}$ & Instruments & $\begin{array}{l}\text { EPIC } \\
\text { Filter }\end{array}$ & $\begin{array}{c}\text { Start Time } \\
\text { y-m-d h:m:s }\end{array}$ & $\begin{array}{c}\text { Stop Time } \\
\text { y-m-d h:m:s }\end{array}$ & $\begin{array}{c}\text { Exposure } \\
{[\mathrm{s}]}\end{array}$ \\
\hline HD 283572 & $21-039$ & 0101440701 & RGS2, MOS1 & Medium & $2000-09-05$ 02:57:44 & $2000-09-05 \quad 15: 47: 55$ & 46211 \\
\hline V773 Tau & $20-042$ & 0203542001 & RGS1, RGS2, MOS1 & Medium & 2004-09-12 07:04:43 & 2004-09-12 15:52:37 & 31674 \\
\hline V410 Tau & $23-032$ & 0086360301 & RGS1, RGS2, MOS1 & Medium & 2001-03-11 12:46:45 & $2001-03-12$ 09:13:24 & 73599 \\
\hline V410 Tau & $24-028$ & 0086360401 & RGS1, RGS2, MOS1 & Medium & 2001-03-12 09:29:38 & 2001-03-12 21:47:51 & 44293 \\
\hline HP Tau/G2 & $08-051$ & 0203540801 & RGS1, RGS2, MOS1 & Medium & $2004-08-2606: 36: 23$ & $2004-08-26$ 18:10:59 & 41676 \\
\hline SU Aur & $26-067$ & 0101440801 & RGS1, RGS2, MOS1 & Thick & 2001-09-21 01:34:17 & 2001-09-22 13:34:31 & 129614 \\
\hline DH Tau & $15-040$ & 0203541501 & RGS1, RGS2, MOS1 & Medium & 2005-02-09 13:12:40 & 2005-02-09 22:38:18 & 33938 \\
\hline BP Tau & $28-100$ & 0200370101 & RGS1, RGS2, MOS1 & Thick & 2004-08-15 06:14:30 & $2004-08-16$ 18:42:57 & 131307 \\
\hline DN Tau & $12-040$ & 0203542101 & RGS1, RGS2, MOS1 & Medium & 2005-03-04 20:22:29 & 2005-03-05 05:01:54 & 31165 \\
\hline AB Aur & 26043 & 0101440801 & RGS1, RGS2, MOS2 & Thick & 2001-09-21 01:34:17 & 2001-09-22 13:34:31 & 129614 \\
\hline
\end{tabular}

${ }^{a}$ XMM-Newton observation identification number.

the task rgsproc. The calibration files used in the data reduction are those described by Pollock (2004). We extracted the total (source+background) spectra and the background spectra separately. In order to optimize the $\mathrm{S} / \mathrm{N}$ of the spectra and given the weakness of most of them, we decided to include only $85 \%$ of the cross-dispersion point-spread function (PSF, $x p s f i n c l=85$ ). For the background extraction, we kept the exclusion region of the cross-dispersion PSF and the inclusion region of the pulseheight distribution at the default values, namely $95 \%$ and $90 \%$ (xpsexcl=95 and pdistincl $=90$ ).

We fitted the spectra in XSPEC (Arnaud 1996), using the optically-thin collisionally-ionized plasma model calculated with the Astrophysical Plasma Emission Code (APEC, Smith et al. 2001). In order to account for calibration discrepancies between the RGS and the MOS detectors, we introduced effective area factors fixed at 1.0 for the MOS and 1.05 for the RGS, applicable to our wavelength region according to Kirsch et al. (2004).

Because we are mainly interested in the line-dominated RGS spectra, we used both RGS spectra (if available) for the fit procedure but only one MOS spectrum (MOS2 for AB Aur and MOS1 for all other stars), the latter confined to short wavelengths (between 1.5 and $9.35 \AA$ ). This range is important for obtaining abundances of $\mathrm{Mg}, \mathrm{Si}, \mathrm{S}$, and Fe. For the RGS spectrum, we used the wavelength region between $8.3 \AA$ and $25.0 \AA$, except for $\mathrm{AB}$ Aur, where we fitted RGS1 between $10 \AA$ and $28 \AA$ and RGS2 between $8.3 \AA$ and $26.5 \AA$, in order to include the $\mathrm{N}$ VII line at $24.78 \AA$ (we chose slightly different wavelength intervals in order to exclude the spectral ranges where an accurate background subtraction was most difficult). A similar approach was followed in Audard et al. (2003) and Telleschi et al. (2005). In order to use the $\chi^{2}$ statistic for the fitting procedure, the total spectra were binned to a minimum of 20 counts per bin for RGS (15 counts per bin for the faint spectrum of DN Tau) and to a minimum of 15 counts per bin for MOS.

The spectra were analyzed using two different approaches. First, we fitted the data with a model consisting of a continuous emission measure distribution (EMD) approximated by two power laws as used in the fits to the EPIC spectra in the XEST survey (Güdel et al. 2007a). The EMD model is approximated by a grid of isothermal components, defining two power laws, one at low temperatures and one at high temperatures. This model was motivated by the EMD shape found from the previous high-resolution X-ray spectroscopy of young solar analog stars (Telleschi et al. 2005). The model can be described by

$Q(T)= \begin{cases}E M_{0} \cdot\left(T / T_{0}\right)^{\alpha}, & \text { for } T \leq T_{0} \\ E M_{0} \cdot\left(T / T_{0}\right)^{\beta}, & \text { for } T>T_{0}\end{cases}$ where $T_{0}$ is the temperature at which the power laws cross, and $E M_{0}$ the emission measure per $\log T$ at this temperature. The slopes of the power laws below and above $T_{0}$ are $\alpha$ and $\beta$, respectively. We left $\beta$ free to vary (between $-3 \leq \beta \leq 1$ ), while we fixed the slope $\alpha$ at a value of 2, as suggested from the EMDs derived in previous studies (Telleschi et al. 2005; Argiroffi et al. 2004). We set low and high temperature cut-offs of the two power laws at $\log T=6.0$ and $\log T=8.0$. The parameters that we fitted are hence $T_{0}, E M_{0}, \beta$ and a selection of elemental abundances.

Although our EMD model is constrained by some approximations (double power-law shape, $\alpha$ fixed at +2 ), for a coronal source it is physically more plausible than a 2 or 3 -temperature model, as the emission is described by a continuous set of temperatures rather than by only 2 or 3 isothermal components. However, because of the approximations, the EMD model could be inadequate if the X-rays were produced, for example, in nearisothermal accretion shocks. Therefore, we went on testing our results with a model with two or three isothermal components. We also applied an EMD model in which $\alpha$ is a free parameter.

In both models, we generally fixed the absorption column density $N_{\mathrm{H}}$ at the value found in the XEST survey from the EPIC data (Table 3 ). These values agree well with $A_{\mathrm{V}}$ measurements from the literature (see Table 1) if a standard gas-to-dust ratio is assumed. We decided to fit $N_{\mathrm{H}}$ only for the high-quality spectrum of SU Aur. The value found in the XEST survey in fact led to problems with some RGS lines, which was not the case when we fitted $N_{\mathrm{H}}$. The $N_{\mathrm{H}}$ found in the fits of SU Aur agrees much better with the $A_{\mathrm{V}}$ values given in the literature $\left(A_{\mathrm{V}}=0.9\right.$ mag, Kenyon \& Hartmann 1995).

We fitted element abundances for the lines that are clearly seen in the spectra $(\mathrm{O}, \mathrm{Ne}, \mathrm{Fe}, \mathrm{Mg}, \mathrm{Si}$, and $\mathrm{S}$; also $\mathrm{N}$ for AB Aur), while we fixed the abundances of those elements that do not show significant features $(\mathrm{C}, \mathrm{N}, \mathrm{Ar}, \mathrm{Ca}, \mathrm{Al}$, and $\mathrm{Ni} ; \mathrm{S}$ for $\mathrm{AB}$ Aur) at the values used in the XEST data analysis $(\mathrm{C}=0.45, \mathrm{~N}=0.788, \mathrm{Ar}=0.55, \mathrm{Ca}=0.195, \mathrm{Al}=0.5$, $\mathrm{Ni}=0.195$, and $\mathrm{S}=0.417$; see Güdel et al. 2007a). All abundances are calculated with respect to the solar photospheric abundances of Anders \& Grevesse (1989) except Fe, for which we used the value given by Grevesse \& Sauval (1999). The above coronal abundances are characteristic of the active, young stars described in the literature (García-Alvarez et al. 2005; Argiroffi et al. 2004). They are arranged according to a weak "inverse First Ionization Potential effect": elements with higher first ionization potential (FIP) are overabundant relative to low-FIP elements if normalized to solar photospheric abundances (Brinkman et al. 2001; Güdel et al. 2001). 
Table 3. Target stars: results from the EPIC spectral fits.

\begin{tabular}{ccccccc}
\hline \hline Stars & $\begin{array}{c}N_{\mathrm{H}} \\
\left(10^{22} \mathrm{~cm}^{-2}\right)\end{array}$ & $\begin{array}{c}T_{0} \\
(\mathrm{MK})\end{array}$ & $\beta$ & $\begin{array}{c}\mathrm{EM}^{1} \\
\left(10^{52} \mathrm{~cm}^{-3}\right)\end{array}$ & $\begin{array}{c}T_{\mathrm{av}} \\
(\mathrm{MK})\end{array}$ & $\begin{array}{c}L_{\mathrm{X}}^{2} \\
\left(10^{30} \mathrm{erg} \mathrm{s}^{-1}\right)\end{array}$ \\
\hline HD 283572 & $0.08(0.07,0.08)$ & $10.4(10.0,10.7)$ & $-0.94(-1.02,-0.87)$ & 114.3 & 14.4 & 13.0 \\
V 773 Tau & $0.17(0.17,0.17)$ & $8.7(8.4,9.1)$ & $-0.87(-0.93,-0.82)$ & 89.8 & 13.0 & 9.5 \\
V 410 Tau & $0.02(0.02,0.03)$ & $10.4(9.4,10.8)$ & $-1.17(-1.28,-0.96)$ & 44.5 & 13.2 & 4.7 \\
HP Tau/G2 & $0.41(0.39,0.42)$ & $9.2(8.6,9.9)$ & $-1.37(-1.46,-1.28)$ & 94.6 & 11.0 & 9.7 \\
SU Aur & $0.47(0.430 .48)$ & $6.4(6.2,7.6)$ & $-1.11(-1.21,-1.06)$ & 95.4 & 8.9 & 9.5 \\
DH Tau & $0.20(0.19,0.21)$ & $11.5(11.0,12.1)$ & $-1.38(-1.49,-1.26)$ & 80.6 & 13.4 & 8.5 \\
BP Tau & $0.06(0.06,0.07)$ & $7.1(6.7,7.6)$ & $-0.67(-0.73,-0.60)$ & 12.8 & 12.3 & 1.4 \\
DN Tau & $0.07(0.07,0.08)$ & $10.5(9.5,11.5)$ & $-1.25(-1.51,-1.02)$ & 11.0 & 12.9 & 1.1 \\
AB Aur & $0.05(0.03,0.07)$ & $4.8(4.3,5.5)$ & $-1.55(-1.78,-1.41)$ & 3.8 & 5.7 & 0.4 \\
\hline
\end{tabular}

${ }^{1}$ Total EM integrated over temperature bins between $\log T=6-7.9$ [K] (see Güdel et al. 2007a for more details).

${ }^{2}$ Calculated in the range $0.3-10 \mathrm{keV}$.

Finally, the X-ray luminosity $L_{\mathrm{X}}$ was determined in the energy range between 0.3 and $10 \mathrm{keV}$ from the best-fit model, assuming a distance of 140 pc (e.g., Loinard et al. 2005; Kenyon et al. 1994).

\section{Results}

In this section, we present the results found from our observations. In Sect. 4.1 we show the light curves, while in Sect. 4.2 we qualitatively describe the spectra. The thermal structures and the element abundances obtained from the spectral fits are presented in Sects. 4.3 and 4.4. Finally, in Sect. 4.5, the X-ray luminosities found here are compared with values found in previous works.

\subsection{Light curves}

Figure 1 shows the light curves of the nine stars. The star V410 Tau was observed twice consecutively (XEST-23 and XEST-24), so both light curves are shown in our figure. For each light curve we also show the hardness, defined as the ratio between the hard band counts (1-4 keV for AB Aur, 2-7.3 keV for all other stars) and the soft band counts $(0.3-1 \mathrm{keV}$ for $\mathrm{AB}$ Aur, 0.3-2 keV for all other stars).

No strong variability (exceeding a factor of two between minimum and maximum in count rate) is seen in the light curves of V773 Tau, HP Tau/G2, and DN Tau. HD 283572 displays slow variability on timescales of $20-30 \mathrm{ks}$. The count rate varies by about $30 \%$. Because the observation lasts about one third of the stellar rotation period, the variation could be due to rotational modulation of X-ray emitting regions in the corona, but equally well due to the evolution of active regions.

DH Tau's brightness decreases during the entire observation. A large flare may have occurred before the observation. Evidence for this hypothesis is the continuously decreasing hardness. Additional support for a flare during the XMM-Newton observation will be discussed in Sect. 4.5.

The light curve of the Herbig star AB Aur also shows variability. We fitted the curve with a sine function and find a modulation with a period of $42.2 \mathrm{~h}$. A similar modulation was also found in the $\mathrm{Mg}$ II lines that are thought to be formed in the wind of AB Aur (see Telleschi et al. 2007 for a detailed discussion).

The two observations of V410 Tau were taken consecutively. The light curve from XEST-23 displays a flare, which has largely decayed in XEST-24. A small flare is visible at about $35 \mathrm{ks}$ in the second light curve. For the spectral fit, we used only the second observation (XEST-24). For a more detailed study of the X-ray variability of this and other TMC X-ray sources, see
Stelzer et al. (2007) and Franciosini et al. (2007). Finally, flares are observed in the light curves of SU Aur and BP Tau. SU Aur exhibits three flares superposed on a slowly decaying light curve. For BP Tau, the total contribution of the flare to the recorded counts is modest, and the quiescent emission contained a sufficient number of counts so that we excluded the flare for the spectral fit. The time interval of the observation ignored for the analysis is marked by the dotted lines (between 15 and $40 \mathrm{ks}$ after the observation start).

\subsection{Spectra}

The EPIC MOS spectra of the nine target stars are shown in Fig. 2. For display purposes, we have multiplied the spectra with different factors (see figure caption). The effect of absorption is clearly visible on the low-energy slopes of the spectra: They are steep for SU Aur and HP Tau/G2, demonstrating high photoelectric absorption, while they are shallow in the other sources. The spectra of HD 283572, SU Aur, and marginally V773 Tau show the Fe line complex at $6.7 \mathrm{keV}$, which is a signature of very hot coronal plasma. We also note the presence of Ly $\alpha$ and He-like lines of $\mathrm{Mg}, \mathrm{Si}$, and $\mathrm{S}$ for these stars. In contrast, the spectrum of AB Aur falls off rapidly above $1 \mathrm{keV}$, suggesting dominance of cool plasma.

Figure 3 shows the fluxed RGS spectra of our sample. The spectra of HD 283572, SU Aur, and HP Tau/G2 reveal a strong continuum clearly pointing to hot plasma. The spectra of SU Aur and HD 283572 show unusually high flux ratios between the strongest Fe lines and the Ne IX or NeX lines when compared with other spectra, suggesting a higher relative Fe abundance in these two stars.

A rather strong $\mathrm{O}$ VII triplet is seen in the spectra of BP Tau, DN Tau, and AB Aur. In the other CTTS spectra, the triplet is not present, probably because the spectra are more absorbed. Despite the low $N_{\mathrm{H}}$ for WTTS, their spectra do not reveal the O VII lines ${ }^{1}$.

\subsection{Thermal structure}

We now present results of our spectral fits. The fitted parameters are listed in Tables 4 and 5 for the EMD model and the 2-T or 3- $T$ model. In Fig. 4 we plot, as an example, the data and the best fit of the EMD model for V773 Tau, but the fits to the

1 The RGS1 was not in use for the observation of HD 283572 and the RGS2 does not cover the O VII triplet due to a CCD chip failure. 

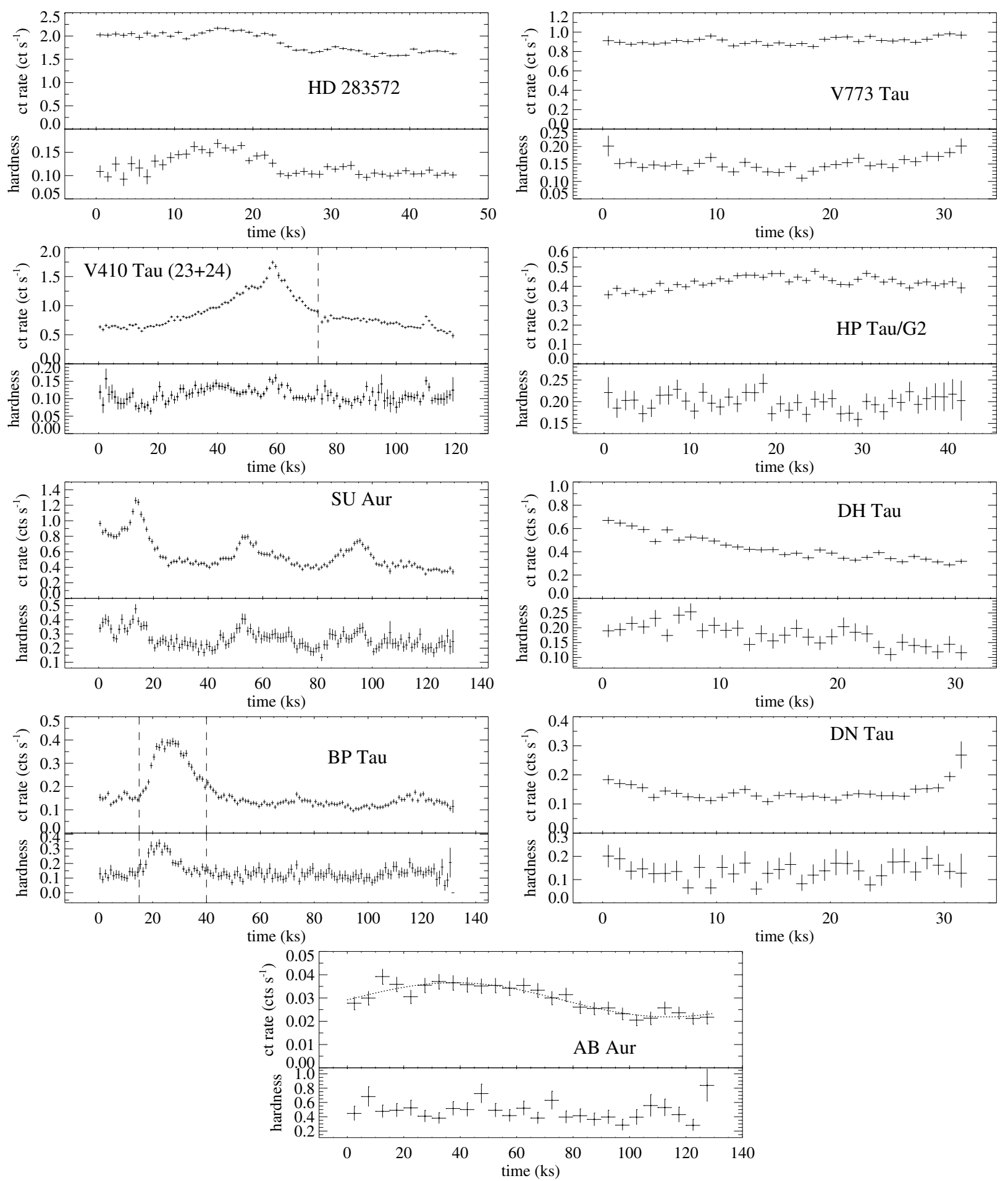

Fig. 1. Light curves of the nine stars. Counts from all EPIC detectors have been combined, except for SU Aur and AB Aur, where data only from the two MOS detectors were available. Binning is to $1 \mathrm{ks}$, except for AB Aur, where $5 \mathrm{ks}$ bins are used. Count rates refer to the energy range of $0.5-7.3 \mathrm{keV}$ (except for $\mathrm{AB}$ Aur, where we used the range $0.3-4 \mathrm{keV}$ ). The size of the crosses in the $x$ direction represent the bin width. Hardness is given by the ratio between the hard band (1-4 keV for AB Aur, 2-7.3 keV for all other stars) and the soft band (0.3-1 keV for AB Aur, 0.3-2 keV for all other stars). The dashed line in the light curve of V410 Tau separates the observations of XEST-23 and XEST-24. The dashed lines in the BP Tau light curve mark the flaring time interval excluded from the spectral fit. The dotted curve overplotted on the AB Aur light curve shows a sinusoidal fit.

other spectra are similar. The reduced $\chi^{2}$ are good for all fits $\left(\chi_{\text {red }}^{2} \lesssim 1.3\right)$ and are very similar for the two approaches.

For each star and each model we computed the average temperature $\left(T_{\text {av }}\right)$ as the logarithmic average of all temperatures used in the fit, applying the emission measures as weights. We see a wide range of thermal properties, from the very hot SU Aur with an average temperature of 20-23 MK, an EMD peaking at about $7.7 \mathrm{MK}$ and a nearly flat high- $T$ slope $(\beta=-0.05)$, to 


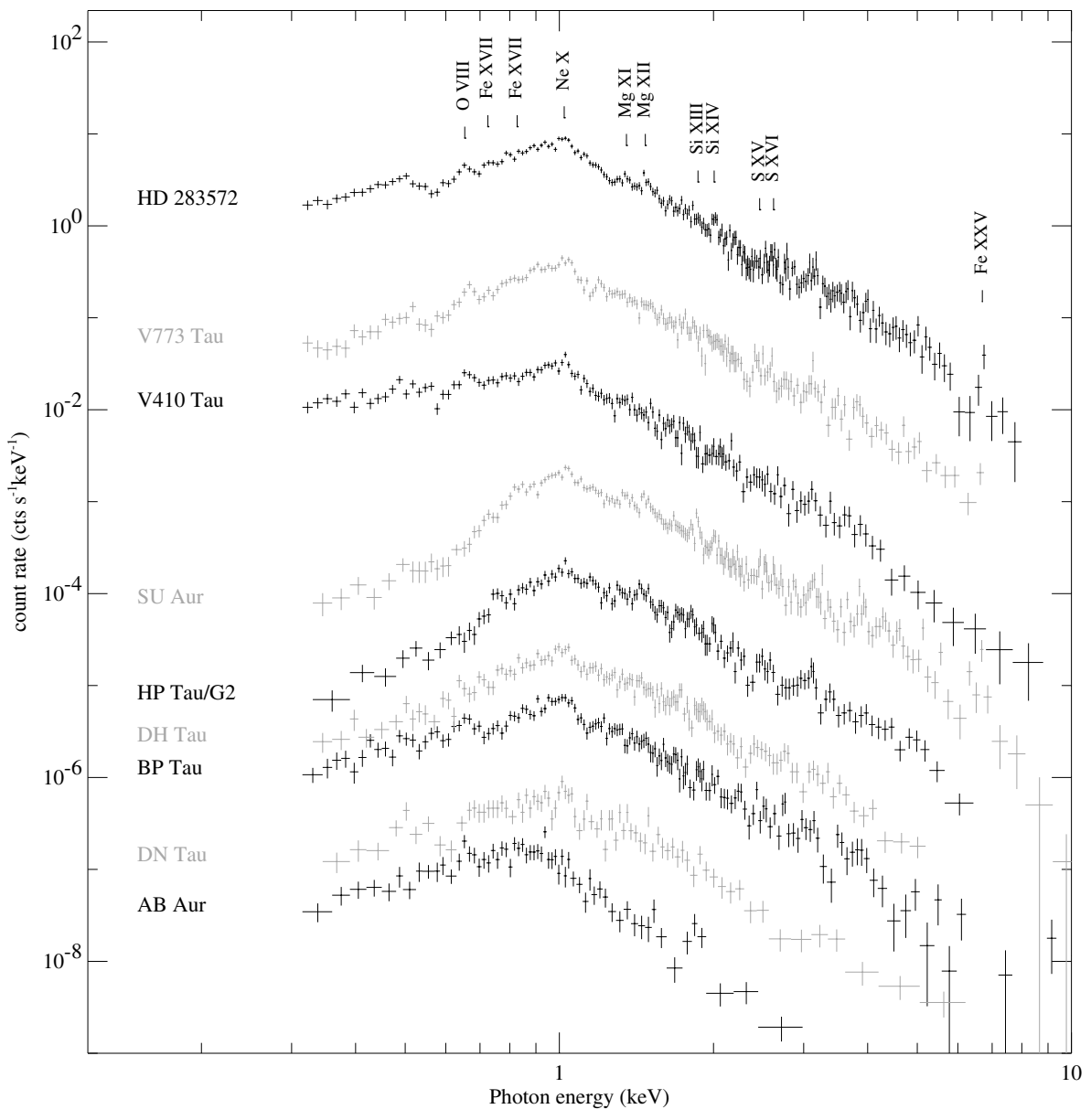

Fig. 2. EPIC MOS1 spectra of the nine target stars. For plotting purposes the spectra have been multiplied with different factors: $10^{-5}$ for DN Tau, $10^{-4}$ for BP Tau and DH Tau, $10^{-3}$ for HP Tau/G2, $10^{-2}$ for SU Aur, $10^{-1}$ for V410 Tau, 1 for V773 Tau, and 10 for HD 283572. the cool $\mathrm{AB}$ Aur, with $T_{\mathrm{av}} \approx 4.7 \mathrm{MK}$ and an EMD peaking at 4.4 MK with a steep high- $T$ slope $(\beta=-1.9)$. For HP Tau/G2, DN Tau, and AB Aur, we fitted a 2-T model instead of the 3-T model because a third component was not required for a good fit.

Adding $\alpha$ as a free fit parameter did not change the abundances significantly, but increased the error bars. The $\chi^{2}$ values were nearly identical. The combination of fit parameters $\alpha$, $\beta$, and $T_{0}$ was ill-constrained for our stellar spectra. This is a consequence of the weakness of our spectra, the small number of lines available, the breadth of the emissivity functions, and the interrelation between emission measure and abundances. We conclude that the EMD fit with fixed $\alpha$ is satisfactory for our spectral analysis.

The average temperatures are similar for the two approaches. The results of the EMD fit are also in quite good agreement with the results from the EPIC fits (Table 3, after Güdel et al. 2007a). Furthermore, our results for SU Aur, BP Tau, and HD 283572 are also within the error ranges of the results reported by Robrade \& Schmitt (2006) and Scelsi et al. (2005).

\subsection{Abundances}

The abundances are listed in Tables 4 and 5, and are shown graphically in Fig. 5 with respect to solar photospheric abundances. For AB Aur the coronal abundances normalized to the AB Aur photospheric values (Acke \& Waelkens 2004) are also shown. The abundance patterns are similar for the two adopted models. The abundances of HD 283572 first decrease for increasing FIP, reaching a minimum around $10 \mathrm{eV}$ (element $\mathrm{S}$ ). For higher FIP, the abundances increase with FIP. A similar trend, although less marked, can be observed for the abundances of HP Tau/G2.

The abundance patterns of V773 Tau, V410 Tau, DH Tau, and BP Tau are consistent with an inverse FIP effect. Similar patterns are observed in active stars and T Tauri stars (Telleschi et al. 2005; Argiroffi et al. 2004). In contrast, the abundance pattern shown by SU Aur is peculiar to this sample, since is reminiscent of a solar-like FIP effect; i.e. elements with low FIP are more abundant than elements with larger FIP.

As suggested above, we find that the Fe abundances are larger in SU Aur, HP Tau/G2, and marginally in HD 283572 than in the rest of the sample. In the extreme case of SU Aur, the Fe abundance amounts to $0.67-0.81$ times the solar photospheric value. Such high Fe abundance values have been reported for relatively inactive stars, while magnetically active stars usually show a strong Fe depletion (Telleschi et al. 2005; Güdel 2004).

The $\mathrm{Ne} / \mathrm{Fe}$ abundance ratio reaches modest values for SU Aur (0.6-0.75), HD 283572 and HP Tau/G2 (1.5), and AB Aur (2.1-2.6). However, for the other T Tauri stars, regardless of their accretion state, the $\mathrm{Ne}$ abundance is $4-6$ times higher than the $\mathrm{Fe}$ abundance. Such high $\mathrm{Ne} / \mathrm{Fe}$ abundance ratios are unusual for main-sequence stars (Telleschi et al. 2005 reported $\mathrm{Ne} / \mathrm{Fe}$ below 2 for their sample of solar analogs), although they are reminiscent of ratios reported for RS CVn 


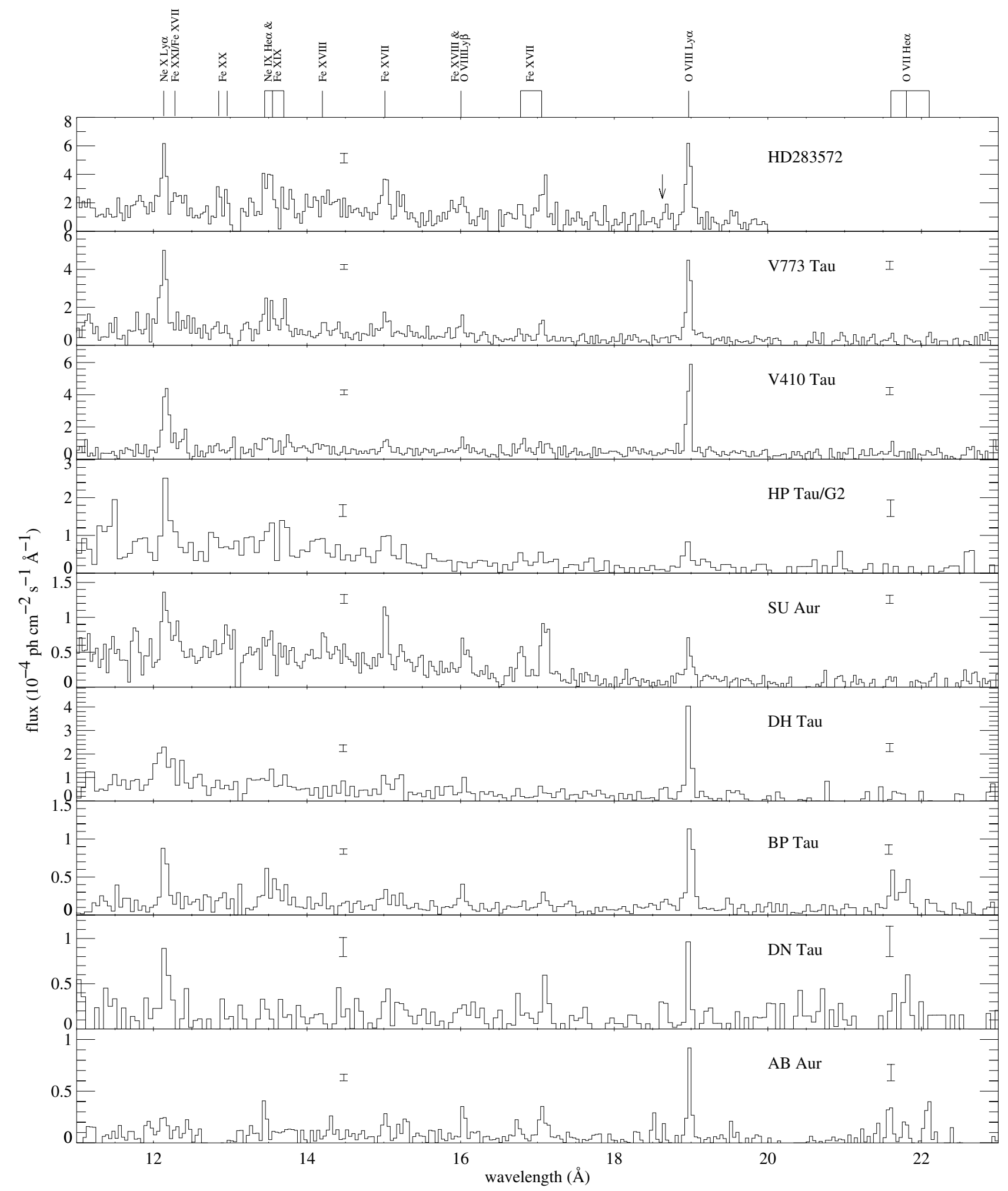

Fig. 3. Co-added and fluxed spectra from the RGS1 and RGS2 instruments. The spectra are background subtracted and are binned to a bin width of $0.066 \AA$ for HP Tau/G2, $0.058 \AA$ for DN Tau and DH Tau, $0.050 \AA$ for BP Tau, $0.042 \AA$ for AB Aur, and $0.035 \AA$ for the other stars. In each spectrum we also plot the typical $1 \sigma$ errors at $14.5 \AA$ and at $21.6 \AA$ (at the position of the O vII line). The arrow in the HD 283572 spectrum indicates the precise wavelength of the O VII He $\beta$ line at $18.63 \AA$.

binaries $(\mathrm{Ne} / \mathrm{Fe}=5.3-13.4$, excluding Capella for which $\mathrm{Ne} / \mathrm{Fe}=0.64$, Audard et al. 2003). A very high $\mathrm{Ne} / \mathrm{Fe}$ abundance ratio has also been measured in TW Hya, reaching values of 7-11 (Kastner et al. 2002; J. Kastner, private communication; Stelzer \& Schmitt 2004; B. Stelzer, private communication; referring to the solar photospheric abundances of Grevesse \& Sauval 1999 for Fe), in TWA 5 ( $\approx 6$, Argiroffi et al. 2005) and in HD $98800(\approx 4$, Kastner et al. 2004, J. Kastner, private communication). For BP Tau, a high $\mathrm{Ne}$ abundance has been reported before $(\mathrm{Ne} / \mathrm{Fe} \approx 5$, Robrade \& Schmitt 2006). 
Table 4. Results from the spectral fits using the EMD model ${ }^{1}$.

\begin{tabular}{|c|c|c|c|c|c|}
\hline Parameters & $\overline{\text { HD } 283572}$ & $\overline{\text { V773 Tau }}$ & V 410 Tau & HP Tau/G2 & \\
\hline$N_{\mathrm{H}}\left[10^{22} \mathrm{~cm}^{-2}\right]$ & $=0.08^{2}$ & $=0.17^{2}$ & $=0.02^{2}$ & $=0.41^{2}$ & \\
\hline$T_{0}[\mathrm{MK}]$ & $10.20(9.20,11.13)$ & $8.72(7.71,9.74)$ & $13.66(10.79,15.17)$ & $10.06(9.07,11.41)$ & \\
\hline $\operatorname{EM}\left[10^{52} \mathrm{~cm}^{-3}\right]^{3}$ & 127.98 & 75.18 & 45.22 & 90.06 & \\
\hline$\beta$ & $-0.84(-0.99,-0.70)$ & $-0.53(-0.67,-0.40)$ & $-1.59(-1.80,-0.87)$ & $-1.15(-1.39,-0.99)$ & \\
\hline $\mathrm{O}^{4}$ & $0.24(0.19,0.29)$ & $0.48(0.39,0.59)$ & $0.39(0.30,0.47)$ & $0.20(0.06,0.38)$ & \\
\hline $\mathrm{Ne}^{4}$ & $0.50(0.40,0.62)$ & $1.30(1.09,1.55)$ & $1.08(0.83,1.31)$ & $0.65(0.43,0.91)$ & \\
\hline $\mathrm{Mg}^{4}$ & $0.51(0.41,0.63)$ & $0.50(0.37,0.65)$ & $0.26(0.15,0.38)$ & $0.51(0.35,0.70)$ & \\
\hline $\mathrm{Si}^{4}$ & $0.25(0.19,0.32)$ & $0.29(0.21,0.39)$ & $0.12(0.04,0.20)$ & $0.21(0.13,0.31)$ & \\
\hline $\mathrm{S}^{4}$ & $0.18(0.08,0.28)$ & $0.64(0.46,0.83)$ & $0.48(0.33,0.65)$ & $0.26(0.12,0.41)$ & \\
\hline $\mathrm{Fe}^{4}$ & $0.33(0.27,0.39)$ & $0.29(0.23,0.36)$ & $0.20(0.16,0.24)$ & $0.43(0.34,0.56)$ & \\
\hline$T_{\mathrm{av}}[\mathrm{MK}]$ & 14.91 & 15.60 & 14.74 & 12.89 & \\
\hline$L_{\mathrm{X}}\left[10^{30} \mathrm{erg} / \mathrm{s}\right]^{5}$ & 13.26 & 8.85 & 4.57 & 9.60 & \\
\hline$\chi_{\mathrm{red}}^{2}$ & 0.94 & 0.94 & 1.24 & 0.92 & \\
\hline d.o.f. & 251 & 208 & 173 & 131 & \\
\hline Parameters & $\overline{\text { SU Aur }}$ & $\overline{\text { DH Tau }}$ & 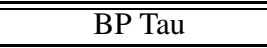 & $\overline{\text { DN Tau }}$ & AB Aur \\
\hline$N_{\mathrm{H}}\left[10^{22} \mathrm{~cm}^{-2}\right]$ & 0.32 & $=0.20^{2}$ & $=0.06^{2}$ & $=0.07^{2}$ & 0.05 \\
\hline$T_{0}[\mathrm{MK}]$ & $7.66(6.95,8.13)$ & $14.77(12.37,17.12)$ & $11.58(10.03,15.11)$ & $6.31(4.16,9.08)$ & $4.38(2.69,5.68)$ \\
\hline $\operatorname{EM}\left[10^{52} \mathrm{~cm}^{-3}\right]^{3}$ & 57.84 & 87.88 & 11.24 & 15.37 & 5.12 \\
\hline$\beta$ & $-0.05(-0.11,0.06)$ & $-2.34(-3.00,-1.73)$ & $-1.21(-2.06,-0.93)$ & $-0.66(-1.06,-0.33)$ & $-1.9(-2.57,-1.52)$ \\
\hline $\mathrm{N}^{4}$ & - & - & - & - & $0.57(0.30,1.13)$ \\
\hline $\mathrm{O}^{4}$ & $0.30(0.24,0.38)$ & $0.49(0.38,0.64)$ & $0.46(0.36,0.59)$ & $0.11(0.05,0.23)$ & $0.22(0.13,0.32)$ \\
\hline $\mathrm{Ne}^{4}$ & $0.38(0.28,0.53)$ & $0.72(0.55,0.94)$ & $0.91(0.66,1.18)$ & $0.45(0.19,0.84)$ & $0.62(0.48,1.04)$ \\
\hline $\mathrm{Mg}^{4}$ & $1.17(1.05,1.43)$ & $0.33(0.20,0.48)$ & $0.50(0.32,0.69)$ & $0.52(0.27,0.90)$ & $0.28(0.13,0.74)$ \\
\hline $\mathrm{Si}^{4}$ & $0.64(0.57,0.79)$ & $0.16(0.07,0.25)$ & $0.26(0.15,0.38)$ & $0.29(0.12,0.56)$ & $0.90(0.60,1.32)$ \\
\hline $\mathrm{S}^{4}$ & $0.57(0.45,0.72)$ & $0.45(0.30,0.62)$ & $0.56(0.34,0.78)$ & $0.11(0.00,0.56)$ & - \\
\hline $\mathrm{Fe}^{4}$ & $0.67(0.61,0.77)$ & $0.16(0.11,0.23)$ & $0.18(0.12,0.24)$ & $0.12(0.06,0.23)$ & $0.29(0.22,0.47)$ \\
\hline$T_{\text {av }}[\mathrm{MK}]$ & 20.07 & 13.76 & 14.28 & 11.30 & 4.71 \\
\hline$L_{\mathrm{X}}\left[10^{30} \mathrm{erg} / \mathrm{s}\right]^{5}$ & 7.79 & 8.20 & 1.16 & 1.24 & 0.39 \\
\hline$\chi_{\text {red }}^{2}$ & 1.23 & 1.00 & 1.32 & 1.06 & 1.02 \\
\hline $\begin{array}{l}\text { ned } \\
\text { d.o.f. }\end{array}$ & 446 & 142 & 161 & 45 & 79 \\
\hline
\end{tabular}

${ }^{1} 68 \%$ error ranges are given in parentheses.

${ }^{2}$ Held fixed at values found in the XEST survey (Güdel et al. 2007a).

${ }^{3}$ Total EM integrated over temperature bins between $\log T=6-7.9$ [K] (see Güdel et al. 2007a for more details).

${ }^{4}$ Element abundances are with respect to solar values given by Anders \& Grevesse (1989) (Grevesse \& Sauval 1999 for Fe).

${ }^{5}$ Determined in the $0.3-10.0 \mathrm{keV}$ band.

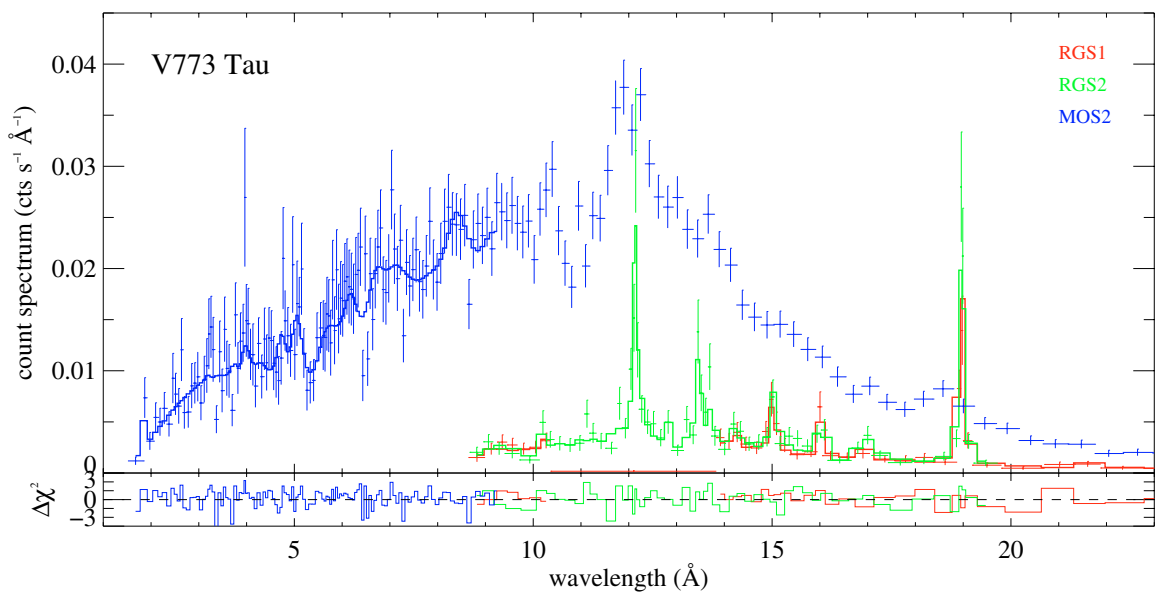

Fig. 4. Data and best-fit spectrum (EMD model) for V773 Tau. The best-fit model is shown by the histograms in the wavelength regions used for the fit.

\subsection{X-ray luminosities}

Large changes in X-ray luminosity of coronal stars are usually accompanied by large changes in spectral parameters (e.g. temperature and densities), in particular in the case of flares. Therefore, it is important to understand if the observed $L_{X}$ is characteristic of the star as judged from previous observations.
The X-ray luminosities from the two methods are in good agreement for all stars.

For HD 283572, $L_{\mathrm{X}} \approx 1.3 \times 10^{31} \mathrm{erg} \mathrm{s}^{-1}$ also agrees well with results found previously by Favata et al. (1998), using other $\mathrm{X}$-ray telescopes $\left(L_{\mathrm{X}}=[0.8-2.1] \times 10^{31} \mathrm{erg} \mathrm{s}^{-1}\right)$, and the value found by Scelsi et al. $\left(2005, L_{\mathrm{X}} \approx 10^{31} \mathrm{erg} \mathrm{s}^{-1}\right.$ ). 
Table 5. Results from the spectral fits using the $2 \mathrm{~T} / 3 \mathrm{~T}$ model ${ }^{1}$.

\begin{tabular}{|c|c|c|c|c|c|}
\hline Parameters & HD 283572 & V773 Tau & V 410 Tau & HP Tau/G2 & \\
\hline$N_{\mathrm{H}}\left[10^{22} \mathrm{~cm}^{-2}\right]$ & $=0.08^{2}$ & $=0.17^{2}$ & $=0.02^{2}$ & $=0.41^{2}$ & \\
\hline$T_{1}[\mathrm{MK}]$ & $2.19(1.33,3.09)$ & $4.51(4.01,5.77)$ & $6.40(3.96,11.28)$ & $8.86(8.43,9.19)$ & \\
\hline$T_{2}[\mathrm{MK}]$ & $8.60(8.33,9.08)$ & $9.15(8.42,10.93)$ & $9.77(5.64,14.17)$ & $25.72(23.42,28.37)$ & \\
\hline$T_{3}[\mathrm{MK}]$ & $26.03(24.96,27.12)$ & $29.39(27.49,32.46)$ & $24.78(22.71,26.69)$ & - & \\
\hline $\mathrm{EM}_{1}\left[10^{52} \mathrm{~cm}^{-3}\right]$ & $18.77(4.50,47.13)$ & $8.01(4.29,16.85)$ & $8.61(4.31,17.16)$ & $28.88(20.95,41.70)$ & \\
\hline $\mathrm{EM}_{2}\left[10^{52} \mathrm{~cm}^{-3}\right]$ & $30.33(26.00,35.88)$ & $20.28(15.56,22.43)$ & $11.25(6.06,18.78)$ & $35.41(31.62,40.19)$ & \\
\hline $\mathrm{EM}_{3}\left[10^{52} \mathrm{~cm}^{-3}\right]$ & $65.79(62.50,69.00)$ & $37.88(34.30,40.89)$ & $23.32(20.61,26.61)$ & - & \\
\hline $\mathrm{O}^{3}$ & $0.27(0.19,0.46)$ & $0.61(0.47,0.79)$ & $0.45(0.37,0.55)$ & $0.51(0.20,0.89)$ & \\
\hline $\mathrm{Ne}^{3}$ & $0.91(0.72,1.12)$ & $1.60(1.29,2.01)$ & $1.10(0.91,1.35)$ & $1.10(0.70,1.56)$ & \\
\hline $\mathrm{Mg}^{3}$ & $0.80(0.65,0.97)$ & $0.57(0.42,0.78)$ & $0.27(0.15,0.39)$ & $0.82(0.56,1.09)$ & \\
\hline $\mathrm{Si}^{3}$ & $0.34(0.25,0.44)$ & $0.30(0.19,0.42)$ & $0.09(0.00,0.18)$ & $0.29(0.17,0.42)$ & \\
\hline$S^{3}$ & $0.22(0.10,0.35)$ & $0.57(0.37,0.78)$ & $0.48(0.30,0.66)$ & $0.27(0.09,0.46)$ & \\
\hline $\mathrm{Fe}^{3}$ & $0.59(0.49,0.68)$ & $0.37(0.27,0.49)$ & $0.18(0.14,0.23)$ & $0.72(0.49,0.97)$ & \\
\hline$T_{\mathrm{av}}[\mathrm{MK}]$ & 12.96 & 16.38 & 14.84 & 15.94 & \\
\hline$L_{\mathrm{X}}\left[10^{30} \mathrm{erg} / \mathrm{s}\right]^{4}$ & 13.56 & 8.75 & 4.60 & 9.26 & \\
\hline$\chi_{\text {red }}^{2}$ & 0.91 & 0.95 & 1.15 & 0.94 & \\
\hline d.o.f. & 248 & 205 & 170 & 130 & \\
\hline Parameters & SU Aur & $\overline{\mathrm{DH} \mathrm{Tau}}$ & BP Tau & $\overline{\text { DN Tau }}$ & AB Aur \\
\hline$N_{\mathrm{H}}\left[10^{22} \mathrm{~cm}^{-2}\right]$ & $0.31(0.30,0.33)$ & $=0.20^{2}$ & $=0.06^{2}$ & $=0.07^{2}$ & $=0.05^{2}$ \\
\hline$T_{1}[\mathrm{MK}]$ & $7.54(7.30,7.75)$ & $4.66(3.84,6.08)$ & $2.97(2.69,3.24)$ & $6.30(5.36,6.77)$ & $2.45(2.10,2.81)$ \\
\hline$T_{2}[\mathrm{MK}]$ & $13.97(12.05,18.06)$ & $11.28(10.27,12.32)$ & $9.42(8.40,10.83)$ & $26.09(21.35,31.66)$ & $6.99(6.62,7.41)$ \\
\hline$T_{3}[\mathrm{MK}]$ & $38.14(35.68,41.14)$ & $24.55(21.36,27.69)$ & $23.59(21.58,25.88)$ & - & - \\
\hline $\mathrm{EM}_{1}\left[10^{52} \mathrm{~cm}^{-3}\right]$ & $11.27(9.18,14.04)$ & $10.85(6.05,16.97)$ & $2.18(1.41,3.28)$ & $4.57(2.77,9.50)$ & $2.11(0.87,3.67)$ \\
\hline $\mathrm{EM}_{2}\left[10^{52} \mathrm{~cm}^{-3}\right]$ & $7.25(4.53,14.35)$ & $37.93(24.16,45.46)$ & $2.84(1.99,4.05)$ & $6.41(5.28,7.97)$ & $3.44(3.06,4.31)$ \\
\hline $\mathrm{EM}_{3}\left[10^{52} \mathrm{~cm}^{-3}\right]$ & $30.49(24.66,33.21)$ & $34.79(25.10,46.39)$ & $6.17(5.36,7.05)$ & - & - \\
\hline $\mathrm{N}^{3}$ & - & - & - & - & $0.45(0.22,0.78)$ \\
\hline $\mathrm{O}^{3}$ & $0.50(0.40,0.61)$ & $0.47(0.32,0.55)$ & $0.30(0.23,0.42)$ & $0.27(0.10,0.41)$ & $0.20(0.16,0.42)$ \\
\hline $\mathrm{Ne}^{3}$ & $0.61(0.46,0.78)$ & $0.69(0.51,0.88)$ & $1.18(0.83,1.58)$ & $1.01(0.44,1.45)$ & $0.60(0.41,0.88)$ \\
\hline $\mathrm{Mg}^{3}$ & $1.51(1.33,1.74)$ & $0.29(0.15,0.44)$ & $0.64(0.42,0.91)$ & $0.77(0.39,1.37)$ & $0.21(0.09,0.38)$ \\
\hline $\mathrm{Si}^{3}$ & $0.79(0.69,0.92)$ & $0.13(0.05,0.23)$ & $0.30(0.16,0.45)$ & $0.41(0.15,0.79)$ & $0.70(0.48,1.01)$ \\
\hline $\mathrm{S}^{3}$ & $0.64(0.49,0.79)$ & $0.44(0.28,0.62)$ & $0.48(0.27,0.72)$ & $0.00(0.00,0.31)$ & - \\
\hline $\mathrm{Fe}^{3}$ & $0.81(0.73,0.91)$ & $0.17(0.11,0.23)$ & $0.27(0.19,0.35)$ & $0.21(0.07,0.37)$ & $0.23(0.17,0.32)$ \\
\hline$T_{\mathrm{av}}[\mathrm{MK}]$ & 22.65 & 13.90 & 12.48 & 14.44 & 4.69 \\
\hline$L_{\mathrm{X}}\left[10^{30} \mathrm{erg} / \mathrm{s}\right]^{4}$ & 7.40 & 8.35 & 1.20 & 1.19 & 0.40 \\
\hline$\chi_{\text {red }}^{2}$ & 1.20 & 1.00 & 1.26 & 0.99 & 1.06 \\
\hline d.o.f. & 443 & 139 & 158 & 44 & 78 \\
\hline
\end{tabular}

${ }^{1} 68 \%$ error ranges are given in parentheses.

${ }^{2}$ Held fixed at values found in the XEST survey (Güdel et al. 2007a).

${ }^{3}$ Element abundances are with respect to solar values given by Anders \& Grevesse (1989) (Grevesse \& Sauval 1999 for Fe).

${ }^{4}$ Determined in the $0.3-10.0 \mathrm{keV}$ band.

For V773 Tau we measured $L_{\mathrm{X}} \approx 8.8 \times 10^{30} \mathrm{erg} \mathrm{s}^{-1}$. This value is slightly higher than was found by Feigelson et al. (1994) in their ROSAT observation $\left(L_{\mathrm{X}}=5.5 \times 10^{30} \mathrm{erg} \mathrm{s}^{-1}\right.$; in the energy range $0.2-2 \mathrm{keV})$. Our $L_{\mathrm{X}}$ is consistent with the quiescent emission measured using ASCA by Skinner et al. (1997), who found $L_{\mathrm{X}}=1.23 \times 10^{31} \mathrm{erg} \mathrm{s}^{-1}$ assuming a distance of $150 \mathrm{pc}$, corresponding to $L_{\mathrm{X}}=1.07 \times 10^{31} \mathrm{erg} \mathrm{s}^{-1}$ at $140 \mathrm{pc}$.

Our $L_{X}$ for V410 Tau $\left(L_{X}=4.6 \times 10^{30} \mathrm{erg} \mathrm{s}^{-1}\right.$ for XEST-24) is lower than the values found in the ROSAT observation by Strom \& Strom $(1994)\left(L_{\mathrm{X}}=1.3 \times 10^{31} \mathrm{erg} \mathrm{s}^{-1}\right)$, while it agrees well with the highest value reported by Stelzer et al. (2003) for a set of recent Chandra observations $\left(L_{\mathrm{X}}=[3.2-4.0] \times\right.$ $10^{30} \mathrm{erg} \mathrm{s}^{-1}$ ). This comparison suggests that the flare seen in the first part of our XMM-Newton observations has largely decayed in the second observation relevant for our study.

The $L_{\mathrm{X}}$ of SU Aur $\left(L_{\mathrm{X}}=[7.4-7.8] \times 10^{30} \mathrm{erg} \mathrm{s}^{-1}\right)$ agrees with the value measured by Robrade \& Schmitt (2006) for the same observation $\left(L_{\mathrm{X}}=8.1 \times 10^{30} \mathrm{erg} \mathrm{s}^{-1}\right)$ and with the value found by Skinner \& Walter $(1998)\left(L_{\mathrm{X}}=[8.4 \pm 0.09] \times 10^{30} \mathrm{erg} \mathrm{s}^{-1}\right)$ in the ASCA observation. For BP Tau, our $L_{\mathrm{X}}\left(\approx 1.2 \times 10^{30} \mathrm{erg} \mathrm{s}^{-1}\right)$ is lower than the value found by Robrade \& Schmitt (2006) $\left(L_{\mathrm{X}}=2.3 \times 10^{30} \mathrm{erg} \mathrm{s}^{-1}\right)$, probably because our $N_{\mathrm{H}}$ is lower and we excluded the flare.

Overall, we find that the long-term variability illustrated by the above comparison is compatible with the short-term variations seen in our light curves. Variations within a factor of $\approx 2$ are common.

The DH Tau light curve is decreasing, suggesting that the source is decaying after a strong flare. To test the activity level of this source, we compared our XMM-Newton observation with ROSAT observations reported in the roshri catalog in $\mathrm{W}_{3} \mathrm{Browse}^{2}$. DH Tau was detected twice by the ROSAT

${ }^{2}$ A service of the Exploration of the Universe Division at NASA/GSFC and the High Energy Astrophysics Division of the Smithsonian Astrophysical Observatory (SAO),

http://heasarc.gsfc.nasa.gov/cgi-bin/W3Browse/ w3browse.pl 

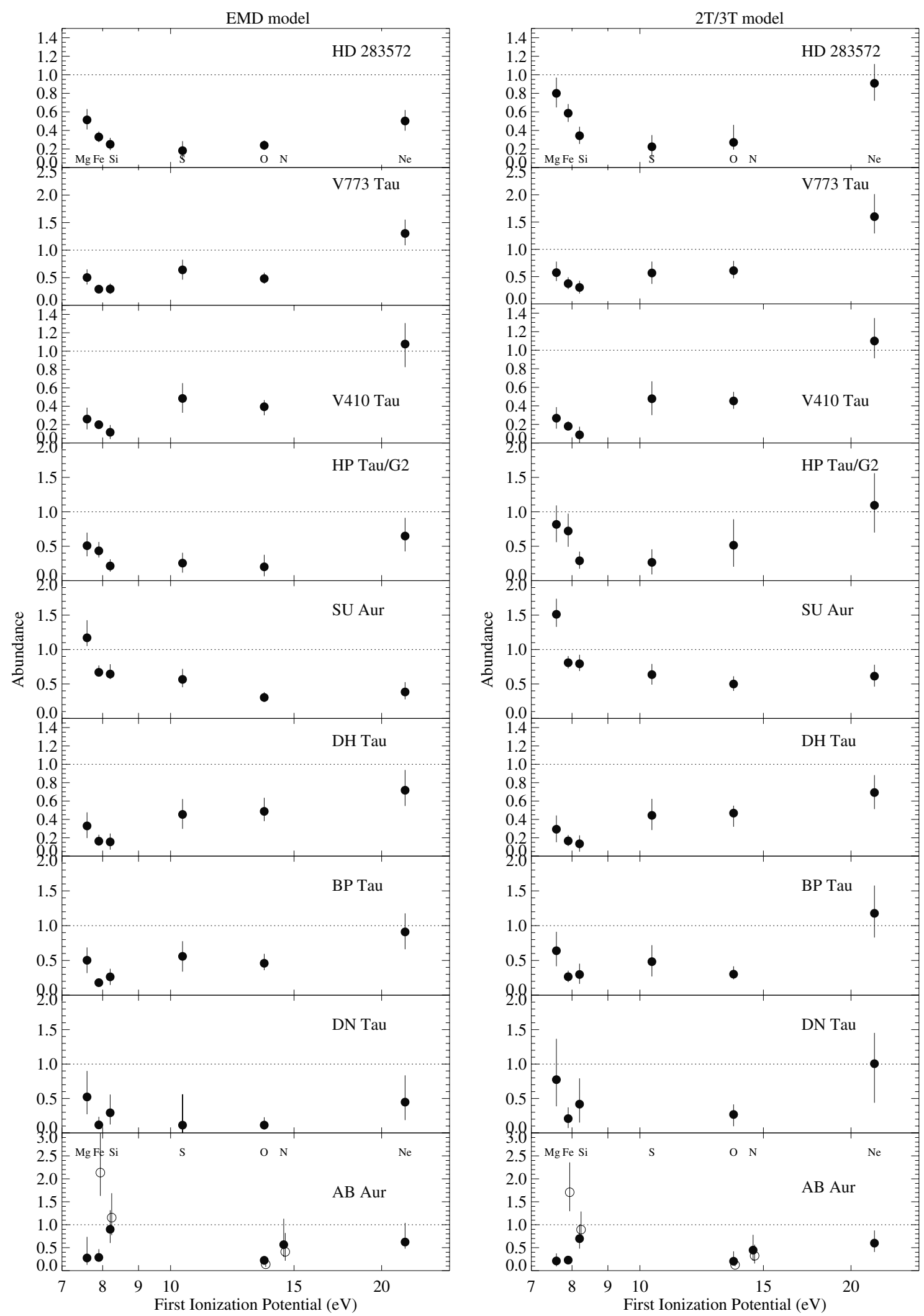

Fig. 5. Element abundances normalized to solar photospheric values (Anders \& Grevesse 1989 and Grevesse \& Sauval 1999 for Fe) derived from the EMD and 3T fits. Open circles for AB Aur: values normalized to the AB Aur photospheric abundances (Acke \& Waelkens 2004).

High-Resolution Imager (HRI) instrument, once with a count rate of $0.020 \mathrm{ct} \mathrm{s}^{-1}$ as 1RXH J042941.3+263256 in observation rh202636, and once as a fainter source in the wings of the nearby
DI Tau (which was much fainter in the first observation) in observation rh201088 (combined source 1RXH J042942.6+263250). In this case, DH Tau was about four times fainter than DI Tau, 
and their total count rate was $0.014 \mathrm{ct} \mathrm{s}^{-1}$. We used PIMMS ${ }^{3}$ to transform count rates to (unabsorbed) fluxes, adopting a temperature of $10 \mathrm{MK}$ and $N_{\mathrm{H}}=2 \times 10^{21} \mathrm{~cm}^{-2}$ (Güdel et al. 2007a) to find $L_{\mathrm{X}}=2.3 \times 10^{30} \mathrm{erg} \mathrm{s}^{-1}$ and $L_{\mathrm{X}}=4 \times 10^{29} \mathrm{erg} \mathrm{s}^{-1}$ in the two observations, i.e., 4-20 times lower than the $L_{X}$ measured in our observation. We conclude that the light curve is the result of the decay of a large flare starting prior to our observation.

\section{The He-like O vil triplet}

The flux ratio of the forbidden $(f)$ line at $22.1 \AA$ and the intercombination $(i)$ line at $21.8 \AA$ of O VII is density-sensitive in the electron-density range between $10^{10} \mathrm{~cm}^{-3}$ and $10^{12} \mathrm{~cm}^{-3}$ (Gabriel \& Jordan 1969). In the case of high densities, the more frequent collisions trigger the excitation from the upper level of the forbidden transition, $1 \mathrm{~s} 2 \mathrm{~s}{ }^{3} \mathrm{~S}_{1}$, to the upper level of the intercombination transitions, $1 \mathrm{~s} 2 \mathrm{p}{ }^{3} \mathrm{P}_{1,2}$. As a consequence, the measured $f / i$ ratio becomes lower. Photoexcitation in an UV radiation field would also decrease the $f / i$ ratio. The photon wavelength for the excitation would correspond to the energy difference of the two upper states, namely $1630 \AA$ for the $\mathrm{O}$ VII triplet. The UV radiation field is thus important for stars with $T_{\text {eff }} \gtrsim 10^{4} \mathrm{~K}$, i.e. only for AB Aur in our sample.

The measured ratio $\mathcal{R}=f / i$ of the forbidden to intercombination line flux can be written as

$\mathcal{R}=\frac{\mathcal{R}_{0}}{1+\phi / \phi_{\mathrm{c}}+n_{\mathrm{e}} / N_{\mathrm{c}}}=\frac{f}{i}$

where $\mathcal{R}_{0}$ is the limiting flux ratio at low densities and for O VII has a value of $\mathcal{R}_{0} \approx 3.85$ (Blumenthal et al. 1972). $N_{\mathrm{c}}$ is the critical density at which $\mathcal{R}=\mathcal{R}_{0} / 2\left(N_{\mathrm{c}} \approx 3.4 \times 10^{10} \mathrm{~cm}^{-3}\right.$ for O VII, Blumenthal et al. 1972). The ratio $\phi / \phi_{\mathrm{c}}$ is the radiation term needed for $\mathrm{AB}$ Aur.

In a thermal plasma, the flux of the resonance line, $r$, is higher than the flux of $f$. Furthermore, for plasma with temperatures higher than $1.5 \mathrm{MK}$, the sum $f+i$ is lower than $r$, so that the "G ratio" $G=(f+i) / r$ is lower than unity (Porquet et al. $2001)$. Given the low $\mathrm{S} / \mathrm{N}$ of our data in the wavelength range of interest, it is difficult to fit the triplet lines individually and simultaneously fulfill the constraints for the $\mathcal{R}$ and $\mathcal{G}$ ratios. We instead proceeded as follows: we used the best-fit results of the 3- $T$ model and then made use of the density-dependent calculations for the O VII line fluxes as implemented in the XSPEC vmekal code. We kept all parameters fixed, except for the electron density and the emission measure of the cooler component to allow for slight adjustments of the total O VII line flux. Thus, the thermal structure intrinsic to the model sets the correct requirement for $\mathcal{G}$ and simultaneously fixes the continuum. The electron density $n_{\mathrm{e}}$ was then varied until a best fit for the fluxes of the O VII $i$ and $f$ lines was found. Only the wavelength region of interest was used for the fit (between 21.4 and $22.3 \AA$ ). The $\mathcal{R}$ ratio was finally measured from the line fluxes.

In Fig. 6 we present the O VII triplet for the stars BP Tau and $\mathrm{AB}$ Aur, the only two stars for which the triplet is clearly visible, together with the best fits and upper or lower limits to the densities. In the spectrum of DN Tau (Fig. 3) an excess of counts is present at the wavelengths of the O VII triplet, but the $\mathrm{S} / \mathrm{N}$ is too low to fit the triplet.

For BP Tau, the background was particularly high near the O VII triplet. We therefore decided to further restrict the inclusion fraction of the cross-dispersion PSF to $70 \%$

\footnotetext{
${ }^{3}$ http://heasarc.gsfc.nasa.gov/Tools/w3pimms.html
}

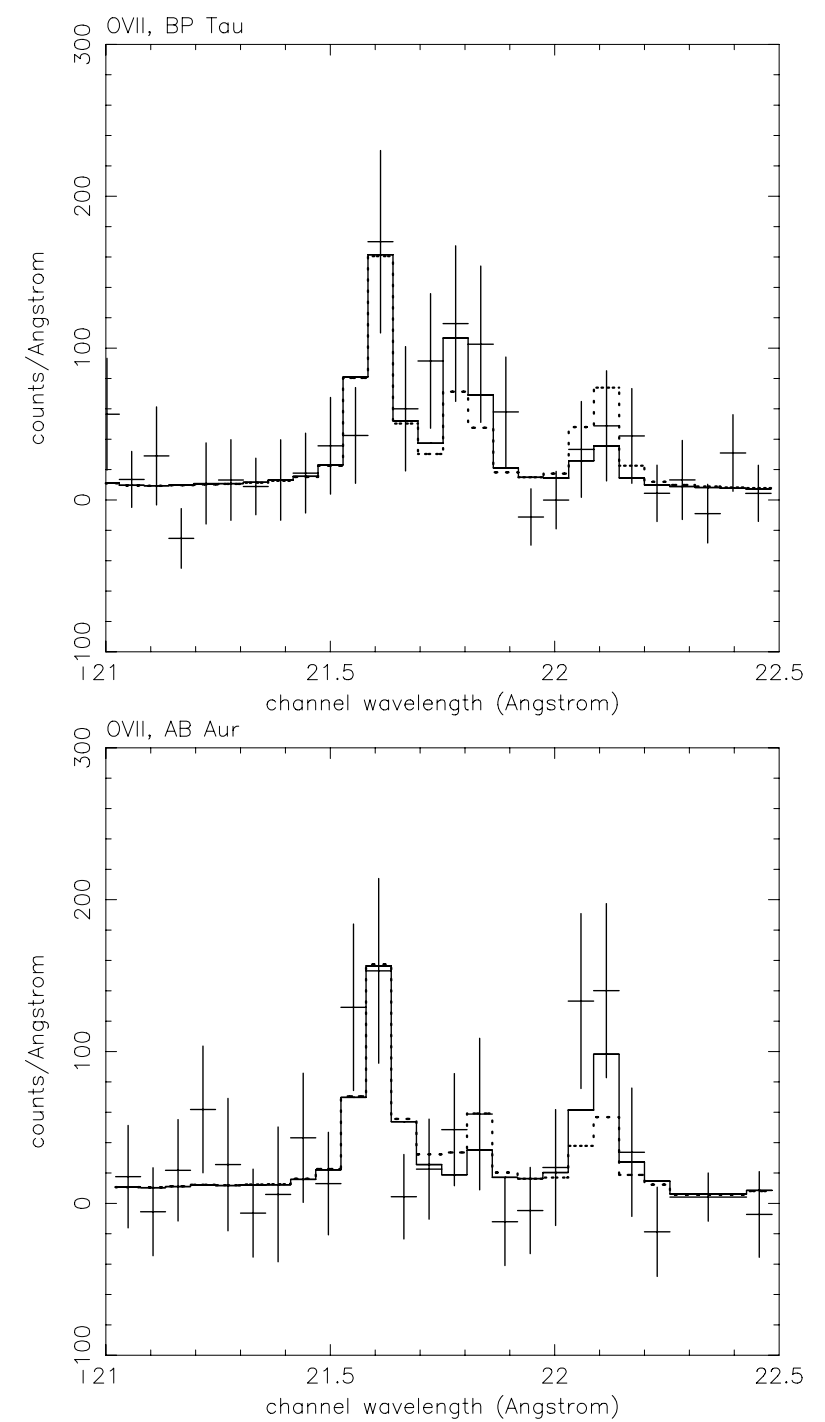

Fig. 6. Fit of the $\mathrm{O}$ VII triplets using variable electron density. Top panel, BP Tau: The best fit for $n_{\mathrm{e}}=3.4 \times 10^{11} \mathrm{~cm}^{-3}$ is plotted as a solid histogram, while the lower density limit $\left(n_{\mathrm{e}}=6.4 \times 10^{10} \mathrm{~cm}^{-3}, 90 \%\right.$ confidence) is shown by the dotted line. Bottom panel, AB Aur: The solid histogram gives the best fit $\left(n_{\mathrm{e}} \lesssim 10^{10} \mathrm{~cm}^{-3}\right)$, while the dotted histogram is for the $90 \%$ upper limit to $n_{\mathrm{e}}\left(n_{\mathrm{e}}<1.3 \times 10^{11} \mathrm{~cm}^{-3}\right)$.

(xpsfincl $=70 \%$, see Sect. 3 ) for this wavelength region. The resulting spectrum thus contains fewer background counts and the triplet appears more clearly, obviously at the cost of some source counts. We also fitted the emission measure of the hottest component. This allows us to adjust the continuum slightly that, because of slight background subtraction inaccuracies, was not accurately represented. We used bins of $56 \mathrm{~m} \AA$ width for the fit. The best fit is represented by a solid histogram in the top panel of Fig. 6. We find a best-fit electron density of $n_{\mathrm{e}}=3.4 \times 10^{11} \mathrm{~cm}^{-3}$, corresponding to $\mathcal{R}=0.23$, with the $90 \%$ lower limit corresponding to $n_{\mathrm{e}, \min , 90}=6.4 \times 10^{10} \mathrm{~cm}^{-3}$ and $\mathcal{R}=1.07$. For the $68 \%$ error we find $n_{\mathrm{e}, \text { min, } 68}=1.6 \times 10^{11} \mathrm{~cm}^{-3}$, corresponding to $\mathcal{R}=0.76$. Given the low flux in the O VII $f$ line, we were unable to constrain upper limits to the densities. The bestfit density agrees with the densities found by Schmitt et al. (2005) and Robrade \& Schmitt (2006), $n_{\mathrm{e}}=3 \times 10^{11} \mathrm{~cm}^{-3}$ and $n_{\mathrm{e}}=3.2_{-1.2}^{+3.5} \times 10^{11} \mathrm{~cm}^{-3}$, respectively.

In the bottom panel of Fig. 6, the O VII triplet of the Herbig star AB Aur is shown. Results for the electron density 
measured in AB Aur are discussed in detail by Telleschi et al. (2007). Here, we report the main results in order to compare them with results from CTTS. We performed the fit again using a bin width of $56 \mathrm{~m} \AA$ to find an electron density below the low-density limit, $n_{\mathrm{e}} \lesssim 10^{10} \mathrm{~cm}^{-3}$ and $\mathcal{R}=\mathcal{R}_{0}$. The dotted line in Fig. 6b corresponds to the $90 \%$ upper limit, which is $n_{\mathrm{e}, \max , 90} \approx(1.3 \pm 0.4) \times 10^{11} \mathrm{~cm}^{-3}$ and $f / i=0.95$. For the $68 \%$ upper limit we found $n_{\mathrm{e}, \max , 68} \approx(4.2 \pm 1.2) \times 10^{10} \mathrm{~cm}^{-3}$, corresponding to $f / i=2.42$.

\section{Discussion}

In this section we discuss the X-ray properties of the stars, test differences between accreting and non-accreting stars, and try to interpret these differences with models for the X-ray emission. In Sect. 6.1 we study the abundance pattern of our stellar sample and compare them to abundances found in active main-sequence stars. In Sect. 6.2, we compare the amount of soft X-ray emission of accreting and non-accreting stars using the O VII/O VIII flux ratio. Finally, in Sect. 6.3, the accretion-shock hypothesis has been tested for BP Tau and AB Aur, the two stars for which we were able to measure the densities.

\subsection{Abundance patterns}

The three stars with spectral type G (HD 283572, SU Aur, HP Tau/G2) have similar properties. They were classified by Herbig \& Bell (1988) as SU Aurigae-like stars, i.e., late type F to $\mathrm{K}$ stars showing an $\mathrm{H} \alpha$ equivalent width smaller than $10 \AA$, weak Ca II emission, very broad absorption lines $\left(v \sin i>50 \mathrm{~km} \mathrm{~s}^{-1}\right)$, and a relatively high optical luminosity.

In X-rays, these stars display an abundance pattern with $\mathrm{Fe}$ enhanced relative to oxygen $(\mathrm{Fe} / \mathrm{O} \approx 1.4$ to 2.2$)$, in contrast to the later-type stars in our sample that show an inverse FIP effect $(\mathrm{Fe} / \mathrm{O} \approx 0.35$ to 1.0$)$. Furthermore, the $\mathrm{Ne} / \mathrm{Fe}$ abundance ratio is relatively small for these three stars $(0.5-1.5)$ if compared with the other $\mathrm{T}$ Tauri stars in our sample $(\mathrm{Ne} / \mathrm{Fe} \approx 4-6)$. In Figs. 7a, b, we plot the $\mathrm{Fe} / \mathrm{O}$ abundance ratio as a function of the $\mathrm{Fe} / \mathrm{Ne}$ ratio for the EMD and $2 \mathrm{~T} / 3 \mathrm{~T}$ models, respectively. The two stellar populations are different: the K-M type stars are lower in both $\mathrm{Fe} / \mathrm{O}$ and $\mathrm{Fe} / \mathrm{Ne}$ than the G-type stars. One possible explanation is that the abundances might be related to mass or to surface gravity. However, then we would expect V410 Tau and V773 Tau to show similar abundance ratios to the G-type stars, as their masses are close to the masses of the SU Aurigaelike stars in our sample, but this is not observed. Scelsi et al. (2005) have also found similar abundances (when scaled to the Fe abundances) for three different G-type stars with different masses at different evolutionary stages (HD 283572, EK Dra, and $31 \mathrm{Com})$. They concluded that surface gravity is not a determining factor for coronal abundance ratios.

In Fig. 7 we also show for comparison the abundance ratios of the TTS CR Cha (Robrade \& Schmitt 2006), TW Hya (labeled “TW Hya1" from Kastner et al. 2002, "TW Hya2" from Stelzer \& Schmitt 2004, and "TW Hya3" from Robrade \& Schmitt 2006), and TWA 5 (Argiroffi et al. 2005). The abundances from these previous works have been converted to the photospheric abundance normalization used here, i.e. Grevesse \& Sauval (1999) for Fe and Anders \& Grevesse (1989) for O and Ne. CR Cha is of spectral type K2, TW Hya is K8, and TWA 5 is M1.5. CR Cha and TW Hya3 were interpreted with a 3T model and are therefore plotted in Fig. 7b, while TW Hya1, TW Hya2, and TWA 5 were interpreted with an EMD method and are
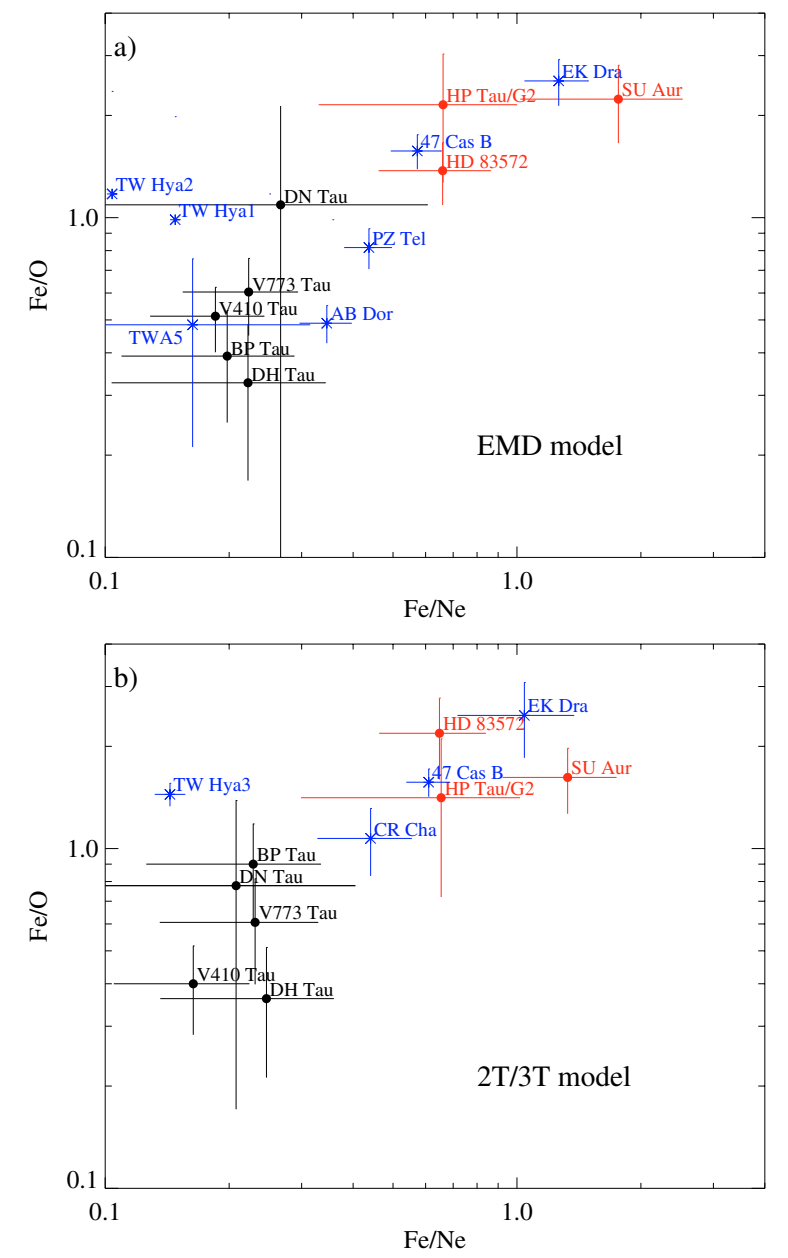

Fig. 7. $\mathrm{Fe} / \mathrm{O}$ abundance ratio as a function of the $\mathrm{Fe} / \mathrm{Ne}$ ratio for abundances derived using an EMD model (a) and a $2 / 3-T$ model (b). The K-M type stars are plotted in black, while the G-type stars are in red. The ZAMS 47 Cas B and EK Dra are plotted in blue for comparison in both plots (from Telleschi et al. 2005). The T Tau star TWA 5, the post T Tau star PZ Tel, the ZAMS AB Dor, TW Hyal and 2 are plotted in (a) for comparison (the analysis were based on an EMD method; see Argiroffi et al. 2005, 2004; García-Alvarez et al. 2005; Kastner et al. 2002; Stelzer \& Schmitt 2004). CR Cha and TW Hya3 have been added to (b) according to $3 \mathrm{~T}$ fits reported by Robrade \& Schmitt (2006).

therefore plotted in Fig. 7a. TWA 5 shows abundance ratios that are compatible with our other K-type TTS, while CR Cha's ratios are somewhat higher. We note, however, that CR Cha is an early K star. TW Hya shows a similar Fe/Ne ratio to other K stars (especially in the measurements from Kastner et al. 2002 and Robrade \& Schmitt 2006), while its Fe/O ratio is high.

A similar abundance pattern to that of SU Aurigae-like stars was also observed in zero-age-main-sequence (ZAMS) stars with spectral type G (47 Cas B, EK Dra, Telleschi et al. 2005). For comparison, we therefore plot in Fig. 7a, b the abundance ratios that were derived from a detailed EMD reconstruction and a multi-thermal fit, respectively, for these two G-type ZAMS stars. All G-type stars display similar abundance ratios independently of the evolutionary stage.

Near zero-age main sequence K stars like PZ Tel (Argiroffi et al. 2004) and AB Dor (Güdel et al. 2001; García-Alvarez et al. 2005) show a classical inverse FIP pattern similar to what we observe in our K-type T Tau stars. The abundance ratios for PZ Tel (Argiroffi et al. 2004) and AB Dor (García-Alvarez et al. 2005) are also shown in Fig. 7a. Again, the abundance ratios are similar 
Table 6. Abundance ratios of active stars from this work and from the literature.

\begin{tabular}{|c|c|c|c|c|c|c|}
\hline Star & Type & Spec.Type & $\mathrm{Fe} / \mathrm{Ne}$ & $\mathrm{Fe} / \mathrm{O}$ & $\mathrm{Ne} / \mathrm{O}$ & ref. \\
\hline \multicolumn{7}{|c|}{ Early to mid G-type stars } \\
\hline HD 283572 & WTTS & G5 & 0.66 & 1.38 & 2.08 & this work \\
\hline HP Tau/G2 & WTTS & G0 & 0.66 & 2.15 & 3.25 & this work \\
\hline SU Aur & CTTS & G2 & 1.76 & 2.23 & 1.27 & this work \\
\hline $47 \mathrm{Cas}$ & ZAMS & G0-2 & 0.57 & 1.57 & 2.74 & Telleschi et al. (2005) \\
\hline EK Dra & ZAMS & G0 & 1.26 & 2.52 & 2.0 & Telleschi et al. (2005) \\
\hline Capella & RS CVn & G1+G8 & 1.56 & 3.13 & 2.00 & Audard et al. (2003) \\
\hline Capella & RS CVn & G1+G8 & 1.41 & 1.65 & 1.16 & Argiroffi et al. (2003) \\
\hline$\sigma^{2} \mathrm{CrB}$ & RS CVn & $\mathrm{G} 1+\mathrm{G} 8$ & 0.73 & 2.15 & 2.94 & Suh et al. (2005) \\
\hline$\sigma^{2} \mathrm{CrB}$ & RS CVn & $\mathrm{G} 1+\mathrm{G} 8$ & 0.69 & 1.82 & 2.66 & Osten et al. (2003) \\
\hline \multicolumn{3}{|c|}{ Averages for G-type stars } & 1.02 & 2.03 & 2.25 & \\
\hline \multicolumn{7}{|c|}{ K-type stars (and early M for TTS) } \\
\hline V773 Tau & WTTS & $\mathrm{K} 2$ & $\overline{0.22}$ & 0.60 & 2.71 & this work \\
\hline V410 Tau & WTTS & K4 & 0.19 & 0.51 & 2.76 & this work \\
\hline DH Tau & CTTS & M1 & 0.22 & 0.33 & 1.47 & this work \\
\hline BP Tau & CTTS & K7 & 0.20 & 0.39 & 1.97 & this work \\
\hline DN Tau & CTTS & M0 & 0.27 & 1.09 & 4.09 & this work \\
\hline CR Cha & CTTS & K2 & 0.44 & 1.07 & 2.42 & Robrade \& Schmitt (2006) \\
\hline TW Hya & CTTS & K8 & 0.14 & 1.44 & 10.06 & Robrade \& Schmitt (2006) \\
\hline TW Hya & CTTS & K8 & 0.15 & 1.00 & 6.67 & Kastner et al. (2002) \\
\hline TW Hya & CTTS & K8 & 0.10 & 1.16 & 11.60 & Stelzer \& Schmitt (2004) \\
\hline TWA 5 & WTTS? & M1.5 & 0.16 & 0.48 & 2.97 & Argiroffi et al. (2005) \\
\hline AB Dor & ZAMS & K0-2 & 0.34 & 0.49 & 1.42 & García-Alvarez et al. (2005) \\
\hline PZ Tel & ZAMS & K0 & 0.44 & 0.81 & 1.87 & Argiroffi et al. (2004) \\
\hline HR 1099 & RS CVn & K1+G5 & 0.15 & 0.36 & 2.40 & Audard et al. (2003) \\
\hline UX Ari & RS CVn & K0+G5 & 0.08 & 0.25 & 3.13 & Audard et al. (2003) \\
\hline VY Ari & RS CVn & K3-4 & 0.14 & 0.45 & 3.22 & Audard et al. (2003) \\
\hline II Peg & RS CVn & K2-3 & 0.07 & 0.14 & 2.02 & Huenemoerder et al. (2001) \\
\hline V851 Cen & RS CVn & K2 & 0.18 & 0.57 & 3.16 & Sanz-Forcada et al. (2004) \\
\hline \multicolumn{3}{|c|}{ Averages for K-type stars } & $0.22^{1}$ & $0.58^{1}$ & $3.00^{1}$ & \\
\hline \multicolumn{7}{|l|}{ Other stars } \\
\hline AB Aur & Herbig & B9.5-A0 & 0.47 & 1.32 & 2.81 & this work \\
\hline AR Lac & RS CVn & $\mathrm{K} 0+\mathrm{G} 2$ & 0.46 & 1.23 & 2.68 & Huenemoerder et al. (2003) \\
\hline$\lambda$ And & RS CVn & G8 & 0.19 & 0.57 & 3.00 & Audard et al. (2003) \\
\hline
\end{tabular}

${ }^{1}$ For TW Hya, the mean of the three abundance ratios was used.

to the K-type TTS, although $\mathrm{Fe} / \mathrm{Ne}$ is somewhat higher. We note, however, that both stars have somewhat earlier spectral types than is typical for our sample of T Tau stars: PZ Tel is classified as a K0 V star (Houk 1978) and AB Dor has spectral type K0-2 V (Vilhu et al. 1987).

Further, we checked the abundance ratios found in active G-type and K-type main-sequence stars in the previous literature using the compilation of Güdel (2004) and a few recent references as given in Table 6 . For these stars as well, both the $\mathrm{Fe} / \mathrm{Ne}$ and $\mathrm{Fe} / \mathrm{O}$ abundance ratios are higher for $\mathrm{G}$ stars than for $\mathrm{K}$ stars. In Table 6 we summarize the abundance ratios for $\mathrm{G}$ and K-type stars. The last three entries in Table 6 are stars that cannot be classified easily. AR Lac is composed of a $\mathrm{G}$ and a $\mathrm{K}$ star and both components contribute strongly to the X-ray emission (Siarkowski et al. 1996). The source $\lambda$ And is a G8 giant star, i.e. intermediate between the two samples, in fact showing similar abundances to K-type stars. Finally, AB Aur is a Herbig star. These last three stars are not included below in the calculations of averages. We do not report the errors because we are mainly interested in studying the distributions, i.e. their means and their standard deviations. Further, the abundances reported in the table originate from different works based on different methods, implying that error estimates may not be consistent with each other. Also, errors are not given in some papers. The mean ratios for $\mathrm{G}$ stars are $\langle\mathrm{Fe} / \mathrm{Ne}\rangle_{\mathrm{G}}=1.02$ (standard deviation $\sigma=0.48$ ) and $\langle\mathrm{Fe} / \mathrm{O}\rangle_{\mathrm{G}}=2.03(\sigma=0.42)$. For $\mathrm{K}$ stars (using the mean of the three abundance ratios for TW Hya), we find $\langle\mathrm{Fe} / \mathrm{Ne}\rangle_{\mathrm{K}}=0.22$ $(\sigma=0.11)$ and $\langle\mathrm{Fe} / \mathrm{O}\rangle_{\mathrm{K}}=0.58(\sigma=0.32)$, i.e. substantially lower than for G-type stars. Considering only the subsample of TTS, we find $\langle\mathrm{Fe} / \mathrm{Ne}\rangle_{\mathrm{G}, \mathrm{TTS}}=1.03$ (standard deviation $\sigma=0.64),\langle\mathrm{Fe} / \mathrm{O}\rangle_{\mathrm{G}, \mathrm{TTS}}=2.92(\sigma=0.47),\langle\mathrm{Fe} / \mathrm{Ne}\rangle_{\mathrm{K}, \mathrm{TTS}}=0.23$ $(\sigma=0.10)$, and $\langle\mathrm{Fe} / \mathrm{O}\rangle_{\mathrm{K}, \mathrm{TTS}}=0.71(\sigma=0.35)$, in agreement with the values found for the full sample. The $\mathrm{Ne} / \mathrm{O}$ abundance ratios are also listed in Table 6 . We find that $\mathrm{Ne} / \mathrm{O}$ ranges between 1 and 3. Drake et al. (2005) suggest that this ratio might be sensitive to accretion; however, we find no difference in the $\mathrm{Ne} / \mathrm{O}$ ratio between CTTS $\left(\langle\mathrm{Ne} / \mathrm{O}\rangle_{\mathrm{C}}=2.24, \sigma_{\mathrm{C}}=1.12\right.$, excluding TW Hya $)$ and WTTS $\left(\langle\mathrm{Ne} / \mathrm{O}\rangle_{\mathrm{W}}=2.75, \sigma_{\mathrm{C}}=0.43\right)$. The mean $\mathrm{Ne} / \mathrm{O}$ ratio for all ( $\mathrm{K}$ and G-type) stars in Table 6 (excluding TW Hya) is $\langle\mathrm{Ne} / \mathrm{O}\rangle=2.76(\sigma=1.65)$. The only star with an exceptionally high $\mathrm{Ne} / \mathrm{O}$ ratio remains TW Hya. This might be related to the older age of TW Hya and the consequent evolution of grains in the disk (Drake et al. 2005). Günther et al. (2006) recently reported an anomalously high $\mathrm{Ne} / \mathrm{O}$ ratio also for the CTTS binary V4046 Sgr.

We conclude that a separation is visible between G-type stars and mid-K-M-type stars, with $G$ stars having a higher $\mathrm{Fe} / \mathrm{Ne}$ abundance ratio. Early K-type stars (like AB Dor, PZ Tel and $\mathrm{CR} \mathrm{Cha}$ ) show an intermediate $\mathrm{Fe} / \mathrm{Ne}$ ratio. A separation also exists in the $\mathrm{Fe} / \mathrm{O}$ abundance ratio if we exclude TW Hya. This star is, however, peculiar among TTS, since almost only cool plasma is present and the abundances essentially refer to 
Table 7. Number of counts and fluences for the O VIII and O VII lines.

\begin{tabular}{|c|c|c|c|c|c|c|c|c|c|}
\hline Star & Line & $\begin{array}{c}\text { Spectrum } \\
\text { used }\end{array}$ & Cts(tot) & Cts(bkg) & Cts(cont) & Cts(line) & $\begin{array}{c}\text { Eff. Area } \\
{\left[\mathrm{cm}^{2}\right]}\end{array}$ & $\begin{array}{l}\text { Fluence } \\
{\left[\mathrm{ph} / \mathrm{cm}^{2}\right]}\end{array}$ & Prob. ${ }^{1}$ \\
\hline \multirow{2}{*}{$\overline{\text { BP Tau }}$} & O VIII & RGS2 & 81 & 10.57 & 11.53 & $58.90 \pm 9.13$ & 52.5 & $1.12 \pm 0.17$ & 0 . \\
\hline & O VII & RGS1 & 80 & 26.26 & 13.44 & $40.30 \pm 9.24$ & 41 & $0.98 \pm 0.23$ & $1.2 \times 10^{-8}$ \\
\hline \multirow[t]{2}{*}{ V773 Tau } & O VIII & RGS2 & 91 & 4.83 & 13.06 & $73.11 \pm 9.59$ & 53 & $1.38 \pm 0.18$ & 0 \\
\hline & O VII & RGS1 & 26 & 5.77 & 11.15 & $9.08 \pm 5.21$ & 41 & $0.22 \pm 0.13$ & $2.4 \times 10^{-2}$ \\
\hline \multirow[t]{2}{*}{ V410 Tau } & O VIII & RGS2 & 82 & 3.61 & 14.78 & $63.61 \pm 9.10$ & 52 & $1.39 \pm 0.20$ & 0. \\
\hline & O VII & RGS1 & 40 & 11.45 & 24.52 & $4.03 \pm 6.51$ & 41 & $0.10 \pm 0.16$ & 0.27 \\
\hline \multirow[t]{2}{*}{ HP Tau } & O VIII & RGS2 & 18 & 5.11 & 2.83 & $10.50 \pm 4.37$ & 53 & $0.20 \pm 0.08$ & $7.8 \times 10^{-4}$ \\
\hline & O VII & RGS1 & 6 & 7.61 & 1.11 & $<2.4$ & 41 & $<0.06$ & 0.87 \\
\hline \multirow[t]{2}{*}{ SU Aur } & O VIII & RGS2 & 59 & 11.37 & 10.36 & $37.27 \pm 7.87$ & 53 & $0.90 \pm 0.19$ & 0. \\
\hline & O VII & RGS1 & 42 & 34.83 & 8.78 & $<6.5$ & 41 & $<0.16$ & 0.62 \\
\hline \multirow{2}{*}{ DH Tau } & O VIII & RGS2 & 67 & 6.59 & 10.02 & $50.39 \pm 8.27$ & 53 & $0.95 \pm 0.16$ & 0. \\
\hline & O VII & RGS1 & 44 & 25.39 & 8.03 & $10.58 \pm 7.62$ & 41 & $0.25 \pm 0.19$ & $4.5 \times 10^{-2}$ \\
\hline \multirow[t]{2}{*}{ DN Tau } & O VIII & RGS1 & 17 & 3.37 & 3.06 & $10.57 \pm 4.21$ & 46 & $0.27 \pm 0.11$ & $3.8 \times 10^{-4}$ \\
\hline & O VII & RGS1 & 20 & 6.66 & 4.32 & $9.02 \pm 4.62$ & 41 & $0.22 \pm 0.11$ & $9.1 \times 10^{-3}$ \\
\hline \multirow[t]{2}{*}{ AB Aur } & O VIII & RGS1 & 54 & 14.57 & 4.05 & $35.38 \pm 7.60$ & 43 & $0.82 \pm 0.18$ & 0. \\
\hline & O VII & RGS1 & 76 & 31.35 & 6.02 & $38.62 \pm 9.16$ & 38 & $0.98 \pm 0.25$ & $2.0 \times 10^{-8}$ \\
\hline \multirow{2}{*}{ HD 283572} & O VIII & RGS2 & $\approx 117$ & - & 33.07 & $83.91 \pm 10.81$ & 52.5 & $1.60 \pm 0.21$ & 0. \\
\hline & O VII & RGS1 & $\approx 55$ & - & 36.51 & $18.18 \pm 7.40$ & 41 & $0.44 \pm 0.18$ & $2.6 \times 10^{-3}$ \\
\hline
\end{tabular}

${ }^{1}$ Probability that the measured number of counts is due to fluctuations in the background and continuum.

this cool plasma, in contrast to other TTS. It therefore seems that the abundance pattern in the coronae of pre-main-sequence and near-ZAMS stars relates to the spectral type, i.e. is a function of the photospheric temperature. We caution that our sample is small, and more studies are needed to consolidate this trend.

\subsection{A soft excess in accreting stars}

In the RGS spectra of Fig. 3, we notice that the weakly absorbed spectra of accreting stars (BP Tau, DN Tau, and AB Aur) display a relatively strong O VII triplet when compared with the O VIII Ly $\alpha$ line. On the contrary, the O VII triplet is not visible in the spectrum of V410 Tau (also subject to weak absorption, $N_{\mathrm{H}}=2 \times 10^{20} \mathrm{~cm}^{-2}$ ). This lets us hypothesize that a substantial cool plasma component is present in the accreting stars but not in the WTTS.

The ratio between the fluxes in the O VII triplet and the $\mathrm{O}$ VIII line varies with temperature in the range of $\approx 1-5 \mathrm{MK}$. In order to estimate this ratio, we derived the number of counts at the wavelengths where the lines are formed (between 18.75-19.2 $\AA$ for O VIII and 21.4-22.2 $\AA$ for O VII). From the total number of counts measured in these wavelength intervals, we subtracted the number of background counts (scaled to the source area) and the number of counts due to continuum (derived from the EMD best-fit model). In the RGS1 the effective area drops substantially at wavelengths slightly shorter than O VIII, and we therefore used the RGS2 spectrum to derive the number of counts in this usually bright line ${ }^{4}$. To obtain the fluence in the lines, we divided the source counts by the effective areas at the relevant wavelengths. The number of counts and the fluences derived with this method are summarized in Table 7. The column heading $\mathrm{Cts}(\mathrm{tot}), \mathrm{Cts}(\mathrm{bkg}), \mathrm{Cts}$ (cont), and $\mathrm{Cts}(\mathrm{src})$ refer to the number of counts measured in the total spectrum, in the background spectrum, in the continuum (computed using the EMD fit results), and in the line (Cts[tot]-Cts[bkg]-Cts[cont]). In the last column, we also list the probability that the measured

${ }^{4}$ We used the RGS1 spectrum only for AB Aur, because in this spectrum the effective area of RGS2 drops at wavelengths close to the O VIII line. number of counts is due to Poissonian fluctuations in the background+continuum. The RGS2 spectrum of DN Tau is difficult to interpret quantitatively because it shows some excess in the background around the O VIII line, resulting in a too low flux in the line. We therefore used the RGS1 to derive the counts in the O VIII line.

The O VII/O VIII fluence (or equivalently, flux) ratio is plotted in Fig. 8 as a function of $N_{\mathrm{H}}$ for our sample and for other objects taken from the literature (Robrade \& Schmitt 2006 for TW Hya, BP Tau, and CR Cha; Argiroffi et al. 2005 for TWA 5). Because the RGS1 was not available and the RGS2 does not cover the $\mathrm{O}$ VII wavelength region, we derived the flux ratio from the best-fit model for HD 283572. Cool temperatures can be determined, apart from the O VII He $\alpha$ line, by the $\mathrm{O}$ VII $\mathrm{He} \beta$ line at $18.63 \AA$, but the line has not significantly been detected. The feature that appears in the HD 283572 spectrum in Fig. 3 is located slightly, but significantly, longward of the O VII $\mathrm{He} \beta$ line (at $18.75 \AA$ instead of $18.63 \AA$ ), while the $\mathrm{O}$ VIII Ly $\alpha$ line is located at its laboratory wavelength. The excess in flux at $18.75 \AA$ in this spectrum is essentially due to a single bin at $18.75 \pm 0.03 \AA$, $2 \sigma$ above the continuum, while the spectral fit represents the data at the correct line wavelength accurately, implying a low flux also for the O VII He $\alpha$ lines. For a further check, we studied a spectrum of HD 283572 observed by Chandra (Audard et al. 2007, in preparation). This spectrum shows a well-developed O VIII line but a line at neither $18.62 \AA$ nor at $18.75 \AA$. The $95 \%$ confidence upper limit for the presence of excess flux at $18.62 \AA$ is approximately $10 \%$ of the $\mathrm{O}$ VIII Ly $\alpha$ flux. Because the emissivity of the O VII $\mathrm{He} \beta$ line is about $14 \%$ of the emissivity of the O VII He $\alpha r$ line at $21.6 \AA$ and the latter is about $60 \%$ of the total triplet flux under typical conditions, we conclude that a flux in the O VII triplet must be lower than O VIII Ly $\alpha$ line flux at the $95 \%$ confidence level. In any case, a strong line at $18.6-18.8 \AA$ as tentatively suggested by the XMM-Newton RGS spectrum can be excluded. The Chandra spectrum shows no indication of flux in the region of the O VII triplet, but the effective area of the HETGS instrument used in this observation is too small to be useful for our study, because even a triplet with a total flux equal to the flux in 


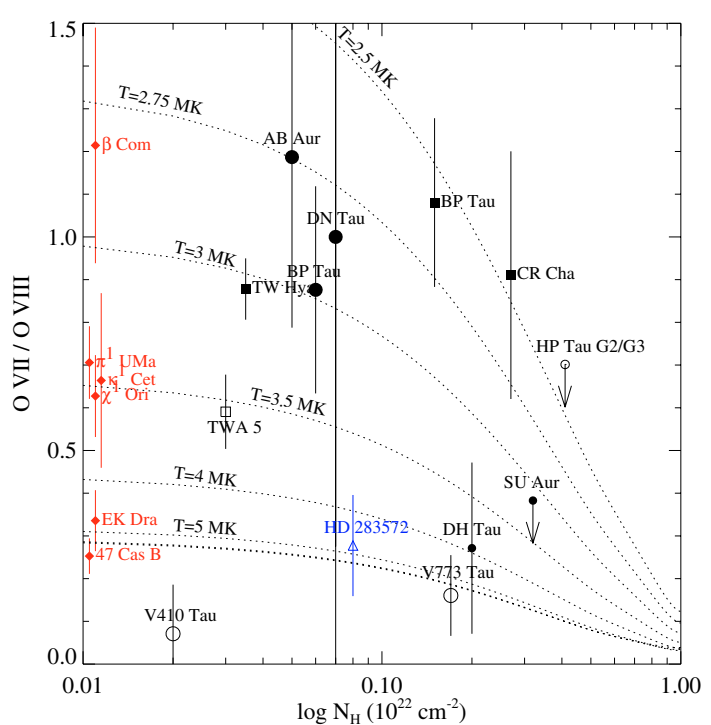

Fig. 8. Ratio of the fluxes measured in the O VII triplet and the O VIII Ly $\alpha$ line as a function of $N_{\mathrm{H}}$. The sample of young stars presented in this paper is plotted with circles, while values taken from the literature are plotted with squares. The diamonds represent the mainsequence solar analogs of Telleschi et al. (2005). HD 283572 is plotted with a triangle because the $\mathrm{O}$ VII line counts are not measured but derived from the best-fit model. Filled symbols denote accreting stars, open symbols are WTTS. Errors are $1 \sigma$. The dotted lines show the theoretical O VII/O VIII ratios for isothermal plasma at a given temperature as a function of $N_{\mathrm{H}}$.

the O VIII Ly $\alpha$ line would not have been detected. For SU Aur and HP Tau/G2, the counts in the O VII triplet were very close to zero, and therefore only $95 \%$ upper limits to the flux ratios are shown. We also note that these stars show the highest $N_{\mathrm{H}}$ in our sample, which is the reason for strong suppression of the O VII triplet. Although the triplet is not explicitly visible in the spectra of V410 Tau, V773 Tau, and DH Tau, we measured a slight excess of counts in the relevant wavelength interval. For these stars, we therefore plot their O VII/O VIII flux ratios at their best-fit loci.

For all WTTS in Fig. 8, we measure a low O VII/O VIII ratio, even if $N_{\mathrm{H}}$ is small $\left(2 \times 10^{20} \mathrm{~cm}^{-2}\right.$ for V410 Tau $)$. The temperatures corresponding to these line ratios $\left(T_{\text {oxy }}\right)$ are consistent with an isothermal plasma of $>3.5 \mathrm{MK}$. The lack of strong O VII is evident in Fig. 3. If the total flux in the O VII were similar to the the flux in the O VIII Ly $\alpha$ line, then the O VII $r$-line would be approximately $50-60 \%$ of the O VIII Ly $\alpha$ flux if $N_{\mathrm{H}}$ is low, which is not seen in Fig. 3 for these WTTS.

For comparison, we also plot the O VII/O VIII ratios of the six solar analog stars presented by Telleschi et al. (2005). These main sequence stars are almost unabsorbed; for illustration purposes, we plot them at $N_{\mathrm{H}} \gtrsim 0.01 \times 10^{22} \mathrm{~cm}^{-2}$. The $\mathrm{O}$ VII/O VIII flux ratios (or the upper limits thereof) that we measure in WTTS compare well with the same ratios measured in active ZAMS stars such as 47 Cas B or EK Dra. With regard to the cool end of the coronal emission measure distribution, WTTS and ZAMS stars seem to behave similarly.

On the other hand, we measure a high O VII/O VIII only for accreting stars. For most of them, the line ratio is consistent with $T_{\text {oxy }} \approx 2.5-3 \mathrm{MK}$. These ratios are reminiscent of $\mathrm{O}$ VII/O VIII found in rather inactive, evolved solar analogs. In contrast to the CTTS, however, the coronae of those more
Table 8. $T_{\text {oxy }}$ derived from Fig. 8 for each star.

\begin{tabular}{lc}
\hline \hline Star & $T_{\text {oxy }}$ \\
\hline BP Tau & $2.97(2.773 .36)$ \\
V773 Tau & $7.0(4.147 .0)$ \\
V410 Tau & $7.0(7.07 .0)$ \\
HP Tau & $>2.36$ \\
SU Aur & $>3.10$ \\
DH Tau & $3.95(3.247 .0)$ \\
DN Tau & $2.84(2.354 .66)$ \\
AB Aur & $2.75(2.473 .11)$ \\
HD 2835721 & $4.51(3.887 .0)$ \\
\hline
\end{tabular}

evolved stars are dominated by cool plasma, while much hotter plasma is common in CTTS.

For two CTTS, namely SU Aur and DH Tau, we measure an $\mathrm{O}$ VII/O VIII ratio lower than for the other accreting stars. However, we noticed that both stars are flaring (see Sect. 4.1), which must lower their O VII/O VIII flux ratio. The O VIII line is sensitive to the hot plasma, so that its flux increases while the source is flaring. In contrast, the O VII triplet is insensitive to the high temperatures measured in a strong flare. We therefore expect the $\mathrm{O}$ VII/O VIII flux ratio to decrease if the source is flaring.

Eventhough $N_{\mathrm{H}}$ does alter the O VII/O VIII ratio, $N_{\mathrm{H}}$ cannot be made responsible for the lack of O VII flux detected in some stars. The range of $N_{\mathrm{H}}$ measured in the WTTS sample is in fact similar to the range measured in CTTS.

According to the $\mathrm{O}$ VII/O VIII ratio measured in the sample investigated here, it is therefore possible that a soft excess is present in all accreting stars. Güdel et al. (2007b) presented the spectrum of the CTTS T Tau. Although the corona of this star is extremly hot, a soft plasma component must be present in order to explain the strong $\mathrm{O}$ VII flux. The flux ratio measured in this star is $\mathrm{O}$ VII/O VIII $=1.06 \pm 0.29$ for $N_{\mathrm{H}}=0.48 \times 10^{22} \mathrm{~cm}^{-2}$. The latter measurements confirm the presence of a soft excess in $\mathrm{T}$ Tau, consistent with the results that we find here. A specific discussion is presented by Güdel et al. (2007b).

In order to illustrate the above trend using physical properties that are not biased by other stellar properties, we derive the temperature $T_{\text {oxy }}$ using the loci for isothermal plasma plotted in Fig. 8. For each star, $T_{\text {oxy }}$ was computed using a spline interpolation. The results are shown in Table 8 , where $T_{\text {oxy }}$ of V410 Tau and V773 Tau are approximately at or below the loci for $7 \mathrm{MK}$, above which the O VII/O VIII ratio is no longer sensitive to temperature. We therefore assign a lower limit for $T_{\text {oxy }}$ of $7 \mathrm{MK}$ to both stars. In Fig. 9, we correlate the $T_{\text {oxy }}$ with stellar accretion parameters.

In the top panel of Fig. 9, we plot $T_{\text {oxy }}$ as a function of the $\mathrm{H} \alpha$ equivalent width. For the stars in the XEST survey, the values of $E W(\mathrm{H} \alpha)$ are taken from Güdel et al. (2007a) and from the references therein (see Table 1). The $E W(\mathrm{H} \alpha)$ values for TW Hya and TWA 5 are from Reid (2003), namely $220 \AA$ and $13.4 \AA$, respectively, while the value for CR Cha is taken from Gauvin \& Strom (1992), namely 43.6 A. For HD 283572, an equivalent width of $0 \AA$ has been reported (Kenyon et al. 1998); we plot an upper limit at $E W(\mathrm{H} \alpha)=1.5 \AA$ for illustration purposes. Generally, we find that stars with a large $E W(\mathrm{H} \alpha)$ show a low $T_{\text {oxy }}$. One problem with using $E W(\mathrm{H} \alpha)$ as an accretion signature is that for the same accretion rate $(\dot{M}), E W(\mathrm{H} \alpha)$ is smaller in G-type stars than in K-type stars due to the formers' high continuum. 

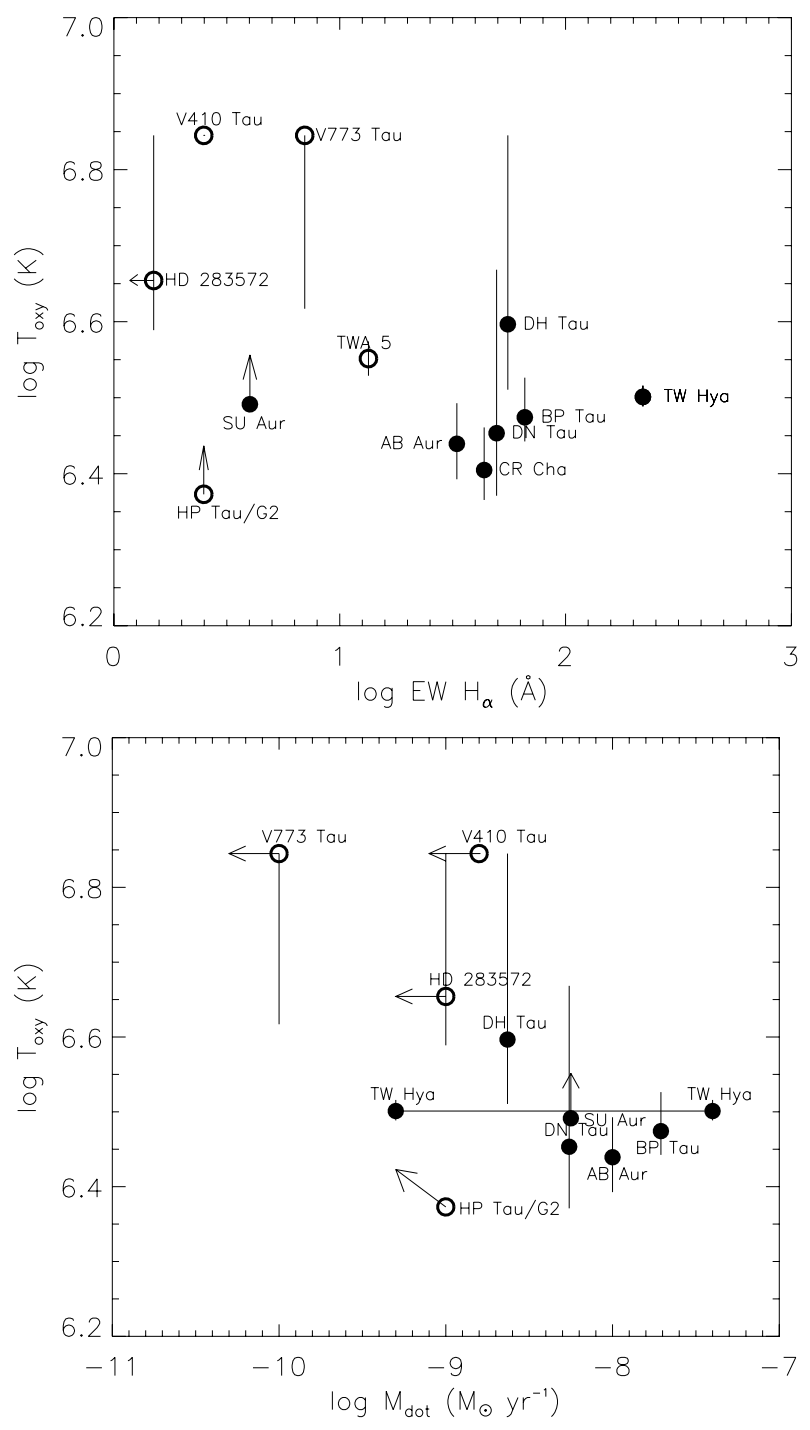

Fig. 9. Temperature corresponding to the observed O VII/O VIII count ratio as a function of $\mathrm{H} \alpha$ equivalent width (top) and as a function of the accretion rate $\dot{M}$ (bottom). Errors in $\log T_{\text {oxy }}$ are $1 \sigma$.

We therefore plot in the bottom panel of Fig. $9 T_{\text {oxy }}$ as a function of $\dot{M}$ (Table 1, Güdel et al. 2007a). In the cases of HD 283572 and HP Tau/G2, for which no upper limit for the accretion rate has been reported, we assign an upper limit of $\dot{M}<10^{-9} M_{\odot} \mathrm{yr}^{-1}$, because for accreting stars with masses similar to HD 283572 and HP Tau/G2, accretion rates between $10^{-9}$ and $10^{-8} M_{\odot} \mathrm{yr}^{-1}$ were clearly identified (Calvet et al. 2004). For V773 Tau and V410 Tau, we used upper limits for the accretion rate as reported in the XEST catalog (see Güdel et al. 2007a for a summary; White \& Ghez 2001). TW Hya is plotted twice, as two different accretion rates have been reported (see Kastner et al. 2002): from $\mathrm{H} \alpha$ measurements, Muzerolle et al. (2000) derived $\dot{M} \approx 5 \times 10^{-10} M_{\odot} \mathrm{yr}^{-1}$, while according to the excess in the C IV $\lambda 1549$ line and the empirical relation between this excess and the accretion rate described by Johns-Krull et al. (2000), $\dot{M}$ could reach values of $(3-6) \times 10^{-8} M_{\odot} \mathrm{yr}^{-1}$. We found no information on an upper limit of $\dot{M}$ for TWA 5 in the published literature, and again recall that this stellar system contains four components.

Despite the low statistics, a trend is clearly visible: stars with low (or vanishing) accretion rates have higher $T_{\text {oxy }}$ than the accreting stars. Observationally, these stars thus reveal a low O VII/O VIII flux ratio.

\subsection{X-rays from accretion?}

Kastner et al. (2002) and Stelzer \& Schmitt (2004) have interpreted the low $f / i$ flux ratio (consistent with high electron density) and other properties of the X-ray spectrum of TW Hya, such as the low X-ray temperature and the low Fe/Ne abundance ratio, in terms of accretion. An analogous scenario has been suggested by Schmitt et al. (2005), Robrade \& Schmitt (2006), and Günther et al. (2006) to explain the low $f / i$ ratio measured in three other stars, BP Tau, CR Cha, and V4046 Sgr. In the last two cases, however, the accretion shock would only be responsible for the softest component of the spectrum, while magnetic activity or star-disk interaction is required to explain the hotter components.

In our stellar sample, an O VII triplet can be measured for only two stars: the CTTS BP Tau and the Herbig star AB Aur. For the two accreting stars SU Aur and DH Tau, the O VII triplet is not present, presumably because it is more absorbed $\left(N_{\mathrm{H}}=\right.$ [3.1-3.2] $\times 10^{21} \mathrm{~cm}^{-2}$ and $N_{\mathrm{H}}=2.0 \times 10^{21} \mathrm{~cm}^{-2}$, respectively) and perhaps also because the $\mathrm{X}$-ray emission was dominated by flaring plasma.

The low $f / i$ flux ratio measured in BP Tau is consistent with high densities $\left(n_{\mathrm{e}}=3.4 \times 10^{11} \mathrm{~cm}^{-3}\right)$. On the other hand, the $f / i$ ratio measured in $\mathrm{AB}$ Aur strictly excludes high densities $\left(n_{\mathrm{e}} \lesssim 10^{10} \mathrm{~cm}^{-3}\right)$.

We now test the accretion hypothesis for BP Tau and AB Aur. The accretion rate of BP Tau is $\dot{M}_{\text {acc }} \approx(1.32-2.88) \times$ $10^{-8} M_{\odot} \mathrm{yr}^{-1}$ (White \& Ghez 2001; Muzerolle et al. 1998). For $\mathrm{AB}$ Aur we approximate the accretion rate as $\approx 10^{-8 \pm 1} M_{\odot} \mathrm{yr}^{-1}$, according to Telleschi et al. (2007) and references therein. The accretion luminosity, assuming that the disk is truncated at the corotation radius $\left(R_{\mathrm{cor}}\right)$, is given by $L_{\mathrm{acc}}=\left(1-R / R_{\mathrm{cor}}\right) G M \dot{M} / R$, which can be written as $L_{\text {acc, } 30} \approx 1200\left(1-\tilde{R} / \tilde{R}_{\text {cor }}\right) \tilde{M} \dot{M}_{-8} / \tilde{R}$, where $L_{\text {acc, } 30}=L_{\mathrm{acc}} /\left(10^{30} \mathrm{erg} \mathrm{s}^{-1}\right), \tilde{M}=M / M_{\odot}, \dot{M}_{\mathrm{acc},-8}=$ $\dot{M}_{\text {acc }} / 10^{-8} M_{\odot} \mathrm{yr}^{-1}, \tilde{R}=R / R_{\odot}$, and $\tilde{R}_{\text {cor }}=R_{\text {cor }} / R_{\odot}$. Using the stellar parameters in Table 1 , we obtain $R_{\mathrm{cor}, \mathrm{BP}} \approx 7.5 R_{\mathrm{BP}}$ and $R_{\mathrm{cor}, \mathrm{AB}} \approx 2 R_{\mathrm{AB}}$ and X-ray luminosities $L_{\mathrm{acc}, \mathrm{BP}} \approx 5 \times 10^{32} \mathrm{erg} \mathrm{s}^{-1}$ and $L_{\mathrm{acc}, \mathrm{AB}} \approx 7 \times 10^{32} \mathrm{erg} \mathrm{s}^{-1}$, i.e. sufficient to account for the observed luminosities.

We further estimate the temperature expected for the accretion shock. The temperature in case of strong shocks is given by $T=3 v^{2} \mu m_{\mathrm{p}} / 16 k$, where the velocity is approximately the free fall velocity $v_{\mathrm{ff}}=(2 G M / R)^{1 / 2}, m_{\mathrm{p}}$ is the proton mass, $k$ the Boltzmann constant, and the mean molecular weight $\mu \approx$ 0.62 for a fully ionized gas. We thus find $T \approx 5.4 \times 10^{6} \tilde{M} / \tilde{R}$ $[\mathrm{K}]$, and with the parameters from Table $1, T_{\mathrm{BP}} \approx 2 \mathrm{MK}$ and $T_{\mathrm{AB}} \approx 6 \mathrm{MK}$. For BP Tau, this temperature is consistent with the temperature of the soft component in the 3-T fit and with the temperature derived from Fig. 8; for $\mathrm{AB}$ Aur, $T_{\mathrm{AB}}$ is consistent with $T_{\text {av }}$.

We can estimate the shock density, using the strong-shock condition $n_{2}=4 n_{1}$, where $n_{1}$ and $n_{2}$ are the pre-shock and postshock densities. The density $n_{1}$ can be estimated from the accretion mass rate and the accreting area on the stellar surface: $\dot{M} \approx 4 \pi R^{2} f v_{\mathrm{fff}} n_{\mathrm{e}} m_{\mathrm{p}}$, where $f$ is the surface filling factor of the accretion flow. We thus find

$n_{2} \approx \frac{4 \times 10^{11}}{\tilde{R}^{3 / 2} \tilde{M}^{1 / 2}} \frac{\dot{M}_{-8}}{f}\left[\mathrm{~cm}^{-3}\right]$ 
According to Calvet \& Gullbring (1998), typical values for $f$ are $f=0.1-10 \%$. The density should then be $n_{2}=10^{12}-10^{14} \mathrm{~cm}^{-3}$ for both stars, given the adopted $\dot{M}$.

These densities are therefore compatible with the O VII triplet fluxes measured in BP Tau, but not with those in $\mathrm{AB}$ Aur. In order to obtain the electron density that we measure in $\mathrm{AB}$ Aur from Eq. (3), $\dot{M}_{\mathrm{AB}}$ should be lowered to about $10^{-10} M_{\odot} \mathrm{yr}^{-1}$; also the accreting area should be at least $10 \%$ and probably more, essentially the whole stellar surface. The first possibility is not supported by the (tentative) measurements of $\dot{M}$ (Telleschi et al. 2007 and references therein), while a filling factor approaching unity is unreasonable, given that the star accretes from a disk and a wind is present (Praderie et al. 1986). We also note that a low $f / i$ ratio has also been measured for the CTTS T Tau (Güdel 2006; Güdel et al. 2007b).

A problem with the accretion scenario is that the shock is formed close to the stellar photosphere (Calvet \& Gullbring 1998) and that the X-rays could therefore be absorbed. Drake (2005) studied the problem for TW Hya. The depth of the shock can be estimated from the measured electron density. For TW Hya the density of $n_{\mathrm{e}} \approx 10^{13} \mathrm{~cm}^{-3}$, derived from Ne IX and O VII triplets, corresponds to a higher $N_{\mathrm{H}}$ than observed. Drake (2005) suggested that $n_{\mathrm{e}}$ is only $\approx 10^{12} \mathrm{~cm}^{-3}$ in TW Hya, while photoexcitation from the ambient $\mathrm{UV}$ radiation field could be responsible for the observed $f / i$ ratio. For BP Tau, we measure a lower electron density than in TW Hya, and we therefore expect that the shock is higher in the photosphere or above the photosphere, and the problem of photospheric absorption is at least in part alleviated, while the influence of the UV radiation field from the shock on the $f / i$ ratio still remains unknown. Accretion can therefore explain the soft excess that we measure in BP Tau, but cannot explain the soft X-ray emission in AB Aur.

\section{Conclusions}

We have presented high-resolution X-ray spectra of nine young stellar objects. Five of them are accreting stars (four CTTS and one Herbig star) and four are WTTS. From previous work on high-resolution X-ray spectroscopy of T Tauri stars (Kastner et al. 2002; Stelzer \& Schmitt 2004; Argiroffi et al. 2005; Schmitt et al. 2005; Robrade \& Schmitt 2006; Günther et al. 2006), three X-ray properties have been proposed to characterize accreting pre-main-sequence stars: i) they show strong soft emission (TW Hya); ii) they display a high electron density (TW Hya, BP Tau, V4046 Sgr); iii) the Ne or $\mathrm{N}$ abundances are relatively high (TW Hya, BP Tau, V4046 Sgr), when compared with Fe. However, the sample of stars that had been studied so far was too small to prove that these three properties are common to all CTTS. Our sample adds a significant number of spectra to test these conjectures.

For two accreting stars (BP Tau and AB Aur), we have been able to measure the $\mathrm{O}$ VII triplet and to derive the source electron density. While we measured a high density for BP Tau $\left(3.4 \times 10^{11} \mathrm{~cm}^{-3}\right.$, confirming previous reports, Schmitt et al. 2005), the density for the Herbig star AB Aur is low, with $n_{\mathrm{e}}<10^{10} \mathrm{~cm}^{-3}$. In the high-resolution X-ray spectrum of the CTTS T Tau (XEST-01-045), the O VII triplet is also consistent with a low density, as reported by Güdel (2006). The low $\mathrm{S} / \mathrm{N}$ does not allow the electron density for the CTTS CR Cha (Robrade \& Schmitt 2006) and DN Tau to be constrained sufficiently well. For the other CTTS, the O VII triplet was not measurable because of strong absorption or, probably, due to the dominance of hot material during strong flaring (in SU Aur and DH Tau).
None of the WTTS in our sample displays an O VII triplet, testifying to the low flux in these lines despite their low absorption and, therefore, pointing to a deficiency of cool material if compared with CTTS. The O VII triplet was previously measured for two other WTTS, TWA 5 (Argiroffi et al. 2005), and HD 98800 (Kastner et al. 2004), and in both cases relatively low densities, reminiscent of the coronae of main-sequence stars, were observed.

In conclusion, apart from several $\mathrm{T}$ Tauri stars for which the O VII remains undetected, there are so far two clear reports each of high (TW Hya, BP Tau) and of low densities (AB Aur, T Tau) in the cooler plasma component. Although high densities are not a condition for qualifying the soft X-ray emission for the accretion scenario, we suggested that the accretion rates and the filling factors would make this scenario unlikely for $\mathrm{AB}$ Aur. A specific discussion of the T Tau observation will be given by Güdel et al. (2007b). The triplet line flux ratios therefore do not seem to be reliable indicators for accretion-induced X-rays on T Tau stars.

As for the issue of overabundances of specific elements such as $\mathrm{Ne}$ or $\mathrm{N}$, we have indeed found the $\mathrm{Ne}$ abundance to be high compared to $\mathrm{Fe}$ (4-6 times higher than the solar ratio), but this is the case for all stars except the G-type stars (SU Aur, HP Tau/G2, and HD 283572) and the Herbig star AB Aur. Stars with a high $\mathrm{Ne}$ abundance in their X-ray source thus comprise both WTTS and CTTS. A high Ne abundance has also been reported for three members of the TW association, namely TW Hya (Kastner et al. 2002), HD 98800 (Kastner et al. 2004), and TWA 5 (Argiroffi et al. 2005), of which the last two seem to be non-accreting $\mathrm{T}$ Tau stars. Therefore, we suggest that the high Ne abundance is not a characteristic property of CTTS, but is common to most young low-mass stars. Studying the abundances of our stellar sample and a sample of other PMS and active MS stars, we have found that the G-type stars on average show abundance ratios of $\langle\mathrm{Fe} / \mathrm{Ne}\rangle_{\mathrm{G}}=1.02$ and $\langle\mathrm{Fe} / \mathrm{O}\rangle_{\mathrm{G}}=2.03$, while we have found significantly lower ratios for K-type stars (on average $\langle\mathrm{Fe} / \mathrm{Ne}\rangle_{\mathrm{K}}=0.22$ and $\langle\mathrm{Fe} / \mathrm{O}\rangle_{\mathrm{K}}=0.58$ ). It thus seems that the abundance ratios are a function of the spectral type and are similar for PMS stars and more evolved active stars, while we find no trend with respect to accretion. The abundance of $\mathrm{N}$ is difficult to measure because the relevant lines are usually rather faint or suppressed by photoelectric absorption. Telleschi et al. (2007) do not find a significant anomaly in AB Aur for which the N VII line could be fitted.

Finally, the outstanding property of the TW Hya spectrum reported previously (Kastner et al. 2002) is the dominance of soft emission relating to cool plasma. Other T Tau stars, however, regularly show hard spectra from which a dominant hot plasma component is inferred (e.g., Preibisch et al. 2005; Güdel et al. 2007a). We have again made specific use here of the highresolution available from the RGS instrument at low photon energies. We have studied the flux ratio of O VII/O VIII that is inaccessible to CCD instruments. The O VII line is formed in a relatively narrow temperature range $(\approx 1-4 \mathrm{MK})$ with the emissivity peaking at $\approx 2 \mathrm{MK}$, whereas the O VIII line forms over a wider range centered at somewhat higher temperatures $(\approx 4 \mathrm{MK})$. The O VII/O VIII flux ratio is thus a good temperature indicator for the plasma at the cool end of the emission measure distribution. The ratio may be modified by photoelectric absorption, but we have corrected for this effect and determined the single temperature that is equivalent to the unabsorbed flux ratio (see Fig. 8). In our sample, it is evident that the accreting stars show an excess in the softest emission (expressed by lower $T_{\text {oxy }}$ in Fig. 8) that is not present in the non-accreting stars. While this soft excess may be of little relevance for the overall emission 
measure distribution dominated by plasma up to $30 \mathrm{MK}$, it does systematically alter the lines formed at the lowest temperatures. The excess of cool emission measure could be due to additional volume containing cool plasma, or else due to increased densities of low-temperature plasma. We cannot conclusively distinguish between these alternatives, but note that accreting stars with high and low densities as inferred from $\mathrm{O}$ VII line ratios have now been reported. We conclude that, while hot plasma may dominate the X-ray sources of most T Tau stars, a soft excess is characteristic of the accreting stars.

What, then, is the mechanism that induces a soft excess in the accreting subsample of our targets? The accretion shock scenario (Kastner et al. 2002) remains a possibility for TW Hya, BP Tau, and V4046 Sgr, although the requirements for AB Aur and $\mathrm{T}$ Tau are rather demanding, as lower accretion rates than hitherto estimated and/or very large accretion areas on the star are required, in contradiction of the standard magnetic-funnel accretion scenario (Calvet \& Gullbring 1998).

Audard et al. (2005) have observed a strongly accreting T Tau star (a so-called EXor object) during an outburst attributed to a strong accretion event. They noted a significant softening of the spectrum during and after outburst, indicating the predominance of cooler plasma, although the X-ray luminosity did not change significantly. They speculated that the accreting material is disrupting the largest magnetic features during outburst, which would also be the hottest if magnetic loops have the same pressure. This would favor emission from smaller, cooler, magnetically-confined regions in those areas where accretion is active.

Alternatively, the accreting material may not disrupt the magnetic structures but may instead fill them with additional cool material that is not driven into the coronal regions from the chromospheric layers by the mechanism of coronal heating. The plasma in the accreting loops is thus cooler from the outset. Also, the increased electron density increases the cooling efficiency of heated loops, because the cooling losses scale as $n_{\mathrm{e}}^{2}$. Magnetic loops loaded with accreting material are therefore cooler (Preibisch et al. 2005), if not otherwise heated preferentially.

Acknowledgements. We thank Beate Stelzer for helpful suggestions and information on TW Hya, and Laurence DeWarf, Edward Fitzpatrick, and Claude Catala for important information on the fundamental properties of AB Aur. We also thank Joel Kastner for information on his TW Hya and HD 98800 spectral analysis. An anonymous referee provided helpful comments that have significantly improved the paper. This research is based on observations obtained with XMM-Newton, an ESA science mission with instruments and contributions directly funded by ESA Member States and the USA (NASA). We would like to thank the International Space Science Institute (ISSI) in Bern, Switzerland, for logistic and financial support during several workshops on the TMC campaign. X-ray astronomy research at PSI has been supported by the Swiss National Science Foundation (grant 20-66875.01 and 20-109255/1). M.A. acknowledges support from NASA grant NNG05GF92G. In addition, he acknowledges support from a Swiss National Science Foundation Professorship (PP002-110504). L.S. acknowledges financial contribution from contract ASIINAF I/023/05/0 and M.I.U.R.

\section{References}

Acke, B., \& Waelkens, C. 2004, A\&A, 427, 1009

Akeson, R. L., Ciardi, D. R., van Belle, G. T., \& Creech-Eakman, M. J. 2002, ApJ, 566, 1124

Anders, E., \& Grevesse, N. 1989, Geochim. Cosmochim. Acta, 53, 197

Argiroffi, C., Maggio, A., \& Peres, G. 2003, A\&A, 404, 1033

Argiroffi, C., Drake, J. J., Maggio, A., et al. 2004, ApJ, 609, 925
Argiroffi, C., Maggio, A., Peres, G., Stelzer, B., \& Neuhäuser, R. 2005, A\&A, 439, 1149

Arnaud, K. A. 1996, in Astronomical Data Analysis Software and Systems V, ed. G. Jacoby, \& J. Barnes (San Francisco: ASP), ASP Conf. Ser., 101, 17

Audard, M., Güdel, M., Sres, A., Raassen, A. J. J., \& Mewe, R. 2003, 398, 1137 Audard, M., Güdel, M., Skinner, S. L., et al. 2005, ApJ, 635, L81

Blumenthal, G. R., Drake, G. W. F., \& Tucker, W. H. 1972, ApJ, 172, 205

Brinkman, A. C., Behar, E., Güdel, M., et al. 2001, A\&A, 365, L162

Calvet, N., \& Gullbring, E. 1998, ApJ, 509, 802

Clavet, N., Muzerolle, J., Briceño, C., et al. 2004, AJ, 128, 1294

Catala, C., Donati, J. F., Böhm, T., et al. 1999, A\&A, 345, 884

DeWarf, L. E., Sepinsky, J. F., Guinan, E. F., Ribas, I., \& Nadalin, I. 2003, ApJ, 590,357

Duchêne, G., Ghez, A. M., McCabe, C., \& Weinberger, A. J. 2003, ApJ, 592, 288

Duvert, G., Guilloteau, S., Ménard, F., Simon, M., \& Dutrey, A. 2000, A\&A, 355,165

den Herder, J. W., Brinkman, A. C., Kahn, S. M., et al. 2001, A\&A, 365, L7

Drake, J. J. 2005, proceeding to the CS 13 conference, ESA SP-560, 519

Drake, J. J., Testa, P., \& Hartmann, L. 2005, ApJ, 627, L149

Favata, F., Micela, G., \& Sciortino, S. 1997, A\&A, 337, 413

Feigelson, E. D., \& DeCampli, W. M. 1981, ApJ, 243, L89

Feigelson, E. D., Casanova, S., Montmerle, T., \& Guibert, J. 1993, ApJ, 416, 623 Feigelson, E. D., Welty, A. D., Imhoff, C., et al. 1994, ApJ, 432, 373

Franciosini, E., Pillitteri, I., Stelzer, B., et al. 2007, 468, 485

Furlan, E., Hartmann, L., Calvet, N., et al. 2006, ApJS, 165, 568

Gabriel, A. H., \& Jordan, C. 1969, MNRAS, 145, 241

García-Alvarez, D., Drake, J. J., Lin, L., Kashyap, V. L., \& Ball, B. 2005, ApJ, 621,1009

Gauvin, L. S., \& Strom, K. M., ApJ, 385, 217

Ghez, A. M., Neugebaure, G., \& Matthews, K. 1993, AJ, 106, 2005

Ghez, A. M., White, R. J., \& Simon, M. 1997, ApJ, 490, 353

Grevesse, N., \& Sauval, A. J. 1999, A\&A, 347, 348

Güdel, M. 2004, A\&ARv, 12, 71

Güdel, M. 2006, Proceedings of the workshop High-resolution X-ray spectroscopy: toward XEUS and Con-X,

http://www.mssl.ucl.ac.uk/gbr/workshop2/papers/guedel_m.pdf

Güdel, M., Audard, M., Briggs, K., et al. 2001, A\&A, 365, L336

Güdel, M., Briggs, K. R., Arzner, K., et al. 2007a, A\&A, 468, 353

Güdel, M., Skinner, S. L., Mel'nikov, S. Yu., et al. 2007b, 468, 529

Günther, H. M., Liefke, C., Schmitt, J. H. M. M., Robrade, J., \& Ness, J.-U. 2006, A\&A, 459, L29

Herbig, G. H. 1960, ApJS, 4, 337

Herbig, G. H., \& Bell, R. K. 1988, Lick Observatory Bulletin, 1111

Houk, N. 1978, Michigan Catalogue of Two-Dimensional Spectral Types for the HD Stars (Ann Arbor: Univ. Michigan)

Huenemoerder, D. P., Canizares, C. R., \& Schulz, N. S. 2001, ApJ, 559, 1135

Huenemoerder, D. P., Canizares, C. R., Drake, J. J., \& Sanz-Forcada, J. 2003, ApJ, 595, 1131

Itoh, Y., Hayashi, M., Tamura, M., et al. 2005, ApJ, 620, 984

Johns-Krull, C. M., Valenti, J. A. \& Linsky, J. L. 2000, ApJ, 539, 815

Kastner, J. H., Huenemoerder, D. P., Schulz, N. S., Canizares, C. R., \& Weintraub, D. A. 2002, ApJ, 567, 434

Kastner, J. H., Huenemoerder, D. P., Schulz, N. S., et al. 2004, ApJ, 605, L49

Kenyon, S. J., \& Hartmann, L. 1995, ApJS, 101, 117

Kenyon, S. J., Dobrzycka, D., \& Hartmann, L. 1994, AJ, 1152491

Kenyon, S. J., Brown, D. I., Tout, C. A., \& Berlind, P. 1998, AJ, 115, 2491

Kirsch, M. G. F., Altieri, B., Chen, B., et al. 2004, EPIC CAL meeting 13, Vilspa, March 2004, ftp://epic3.xra.le.ac.uk/pub/cal-pv/meetings/ vilspa-2004-03/kirsch/04_03_22_vilspa_crosscal_MK.pdf

Koresko, C. D., Herbst, T. M., \& Leinert, C. 1997, ApJ, 480, 741

Leinert, C., Zinnecker, H., Weitzel, N., et al. 1993, A\&A, 278, 129

Loinard, L., Mioduszewski, A. J., Rodríguez, L. F., et al. 2005, ApJ, 619, L179

Mohanty, S., Jayawardhana, R., \& Barrado y Navascués, D. 2003, ApJ, 593, L109

Muzerolle, J., Hartmann, L., \& Calvet, N. 1998, AJ, 116, 2965

Muzerolle, J., Calvet, N., Briceño, C., Hartmann, L., \& Hillenbrand, L. 2000, ApJ, 535, L47

Muzerolle, J., Calvet, N., Hartmann, L., \& D’Alessio, P. 2003a, ApJ, 597, L149

Muzerolle, J., Hillenbrand, L., Calvet, N., Briceño, C., \& Hartmann, L. 2003b, ApJ, 592, 266

Ness, J.-U., \& Schmitt, J. H. 2005, A\&A, 444, L41

Ness, J.-U., Güdel, M., Schmitt, J. H., M. M., Audard, M., \& Telleschi, A. 2004, A\&A, 427, 667

Neuhäuser, R., Sterzik, M. F., Schmitt, J. H. M. M., Wichmann, R., \& Krautter, J. 1995, A\&A, 297, 391

Osten, R. A., Ayres, T. R., Brown, A., Linsky, J. L., \& Krishnamurthi, A. 2003, ApJ, 582,1073 
Pollock, A. M. T. 2004, on behalf of the RGS consortium 2004, Status of the RGS calibration, Doc-id: XMM-SOC-CAL-TN-0030 Issue 3, available at: http://xmm.vilspa.esa.es/docs/documents/

CAL-TN-0030-3-0.ps.gz

Porquet, D., Mewe, R., Dubau, J., Raassen, A. J. J., \& Kaastra, J. S. 2001, A\&A, 376, 1113

Praderie, F., Catala, C., Simon, T., \& Boesgaard, A. M. 1986, ApJ, 303, 311

Preibisch, T., Kim, Y.-C., Favata, F., et al. 2005, ApJS, 160, 401

Ressler, M. E., \& Barsony, M. 2001, AJ, 12, 1098

Reid, N. 2003, MNRAS, 342, 837

Richichi, A., Leinert, Ch., Jameson, R., \& Zinnecker, H. 1994, A\&A, 287, 145

Robrade, J., \& Schmitt, J. H. M. M. 2006, A\&A, 449, 737

Sanz-Forcada, J., Favata, F., \& Micela, G. 2004, A\&A, 416, 281

Scelsi, L., Maggio, A., Peres, G., \& Pallavicini, R. 2005, A\&A, 413, 643

Schmitt, J. H. M. M., Robrade, J., Ness, J.-U., Favata, F., \& Stelzer B. 2005 , A\&AL, 432L, 35

Smith, R. K., Brickhouse, N. S., Liedhal, D. A., \& Raymond, J. C. 2001, ApJ, 556, L91

Siarkowski, M., Pres, P.. Drake, S. A., White, N. E., \& Singh, K. 1996, ApJ, 473, 470
Siess, L., Dufour, E., \& Forestini, M. 2000, A\&A, 358, 593 Skinner, S. L., \& Walter, F. M. 1998, ApJ, 509, 761

Skinner, S. L., Güdel, M., Koyama, K., \& Yamauchi, S. 1997, ApJ, 486, 886 Stelzer, B., \& Neuhäuser, R. 2001, A\&A, 377, 538

Stelzer, B., \& Schmitt, J. H. M. M. 2004, A\&A, 418, 687

Stelzer, B., Fernández, M., Costa, V. M., et al. 2003, A\&A, 411, 517

Stelzer, B., Flaccomio, E., Briggs, K., et al. 2007, 468, 463

Strom, K. M., \& Strom, S. E. 1994, ApJ, 424, 237

Suh, J. A., Audard, M., Güdel, M., \& Paerels, F. B. S. 2005, ApJ, 630, 1074

Telleschi, A., Güdel, M., Briggs, K., et al. 2005, ApJ, 622, 653

Telleschi, A., Güdel, M., Briggs, K. R., et al. 2007, 468, 541

Telleschi, A., Güdel, M., Briggs, K. R., Audard, M., \& Palla, F. 2007, 468, 425

Testa, P., Drake, J. J., \& Peres, G. 2004, ApJ, 617, 508

Turner, M. J. L., Reeves, J. N., Ponman, T. J., et al. 2001, A\&A, 365, L27

Vilhu, O., Gustafsson, B., \& Edvardsson, B. 1987, ApJ, 320, 850

Vuong, M. H., Montmerle, T., Grosso, N., et al. 2003, A\&A, 408, 581

Walter, F. H., \& Kuhi, L. V. 1981, ApJ, 250, 254

Welty, A. D. 1995, AJ, 110, 776

White, R. J., \& Ghez, A. M. 2001, ApJ, 556,265 